\title{
Planck-LFI: Design and Performance of the 4 Kelvin Reference Load Unit
}

\author{
L. Valenziano ${ }^{1}$, F. Cuttaia ${ }^{1}$, A. De Rosa ${ }^{1}$, L. Terenzi ${ }^{1}$, A. Brighenti ${ }^{1}$, G.P. Cazzola ${ }^{1}$, A. Garbesi ${ }^{2}$, S. \\ Mariotti $^{3}$, G. Orsi ${ }^{1}$, L. Pagan ${ }^{5}$, F. Cavaliere ${ }^{6}$, M. Biggi ${ }^{4}$, R. Lapini ${ }^{4}$, E. Panagin ${ }^{4}$, P. Battaglia ${ }^{4}$, R.C. Butler ${ }^{1}$, \\ M. Bersanelli ${ }^{6}$, O. D'Arcangelo ${ }^{7}$, S. Levin ${ }^{8}$, N. Mandolesi ${ }^{1}$, A. Mennella ${ }^{6}$, G. Morgante ${ }^{1}$, G. Morigi ${ }^{1}$, M. \\ Sandri $^{1}$, A. Simonetto ${ }^{7}$, M. Tomasi ${ }^{9}$, F. Villa ${ }^{1}$, M. Frailis ${ }^{10}$, S. Galeotta ${ }^{11}$, A. Gregorio ${ }^{10,11}$, R. Leonardi ${ }^{12}$, \\ S.R. Lowe ${ }^{13}$, M. Maris ${ }^{10}$, P. Meinhold ${ }^{12}$, L. Mendes ${ }^{14}$, L. Stringhetti ${ }^{1}$, A. Zonca ${ }^{9}$, and A. Zacchei ${ }^{10}$ \\ 1 Istituto di Astrofisica Spaziale e Fisica Cosmica - Bologna, INAF, via P. Gobetti, 101 - I40129 Bologna, Italy \\ 2 Istituto per la Sintesi Organica e la Fotoreattività, CNR, via P. Gobetti, 101 - I40129 Bologna, Italy \\ 3 Istituto di Radioastronomia, INAF, via P. Gobetti, 101 - I40129 Bologna, Italy \\ 4 Officine Pasquali, via Palazzo de' Diavoli 124B, - I50142 Firenze, Italy \\ 5 Thales Alenia Space Italia, Sede di Milano, S.S. Padana Superiore, 290 - I20090 Vimodrone, Italy \\ ${ }^{6}$ Università degli Studi di Milano, Via Celoria 16, - I20133 Milano, Italy \\ 7 Istituto di Fisica del Plasma, CNR, Via Roberto Cozzi, 53 - I20125 Milano, Italy \\ 8 Jet Propulsion Laboratory, 4800 Oak Grove Drive, CA91109 - Pasadena, USA \\ 9 Istituto di Astrofisica Spaziale e Fisica Cosmica - Milano, INAF, via E. Bassini, 15 - I20133 Milano, Italy \\ 10 Osservatorio Astronomico di Treste, INAF, via G.B. Tiepolo, 11 - I34143 Trieste, Italy \\ 11 Universitá degli studi di Trieste, Dipartimento di Fisica, via A. Valerio, 2 - I34127 Trieste, Italy \\ 12 Department of Physics, University of California, Santa Barbara 2225 Broida Hall - CA 93106 Santa Barbara, USA \\ 13 Jodrell Bank Centre for Astrophysics, Alan Turing Building, The University of Manchester, Manchester, M13 9PL, \\ UK \\ 14 ESA - European Space Agency, Keplerlaan 1, NL 2200 AG Noordwijk, The Netherlands
}

Received June 23,2009; accepted August 5, 2009

\begin{abstract}
The LFI radiometers use a pseudo-correlation design where the signal from the sky is continuously compared with a stable reference signal, provided by a cryogenic reference load system. The reference unit is composed by small pyramidal horns, one for each radiometer, 22 in total, facing small absorbing targets, made of a commercial resin ECCOSORB $\mathrm{CR}^{\mathrm{TM}}$, cooled to $\sim 4.5 \mathrm{~K}$. Horns and targets are separated by a small gap to allow thermal decoupling. Target and horn design is optimized for each of the LFI bands, centered at 70, 44 and $30 \mathrm{GHz}$. Pyramidal horns are either machined inside the radiometer $20 \mathrm{~K}$ module or connected via external electro-formed bended waveguides. The requirement of high stability of the reference signal imposed a careful design for the radiometric and thermal properties of the loads. Materials used for the manufacturing have been characterized for thermal, RF and mechanical properties. We describe in this paper the design and the performance of the reference system.
\end{abstract}

Key words. experimental cosmology - CMB - space instrumentation - calibrators - cryogenic systems - ECCOSORB

\section{Introduction}

Remark to the $\mathrm{ARX}_{\mathrm{I}} \mathrm{V}$ version

This is an author-created, un-copyedited version of an article published in JINST.IOP Publishing Ltd is not responsible for any errors or omissions in this version of the manuscript or any version derived from it.The present version is derived from the latest version of the paperbefore final acceptance from JINST, thus itcould have some minor differences in phrasing, spelling and style with respect to the published version. The definitive publisher authenticated version is available online at:

http://www.iop.org/EJ/journal/-page=extra.proc5/jinst

Reference : 2009 JINST 4 T12006

DOI: $10.1088 / 1748-0221 / 4 / 12 / \mathrm{T} 12006$

Send offprint requests to: L. Valenziano e-mail: valenziano@iasfbo.inaf.it
Planck (Tauber 2001; Mandolesi et al. 2002b; Puget 2004; Tauber 2005, 2006) is the third generation mission devoted to produce the ultimate image the Cosmic Microwave Background (CMB) anisotropies. Planck will be placed on a Lissajou orbit around the second Lagrangian point of the Earth-Sun system. By following our planet in its revolution motion, it will always be oriented in the anti-Sun direction, spinning around its z-axis at 1 r.p.m. The telescope, whose line-of-sight is at $\sim 90 \mathrm{deg}$ from the spin axis, will allow all detectors in the focal plane to scan almost the whole sky in six months.

Two instruments share the focal surface of a 1.5 mirror off-axis telescope (Mandolesi et al. 2000; Tauber et al. 2009): the High Frequency Instrument (HFI) (Lamarre et al. 2003a,b, 2009) and the Low Frequency Instrument (LFI) (Villa et al.|2002; Mandolesi et al. 2002a; Mennella et al. 2004; Sandri et al.|2004; Valenziano et al. 2007; Mandolesi et al. 2009; Bersanelli et al. 2009). Planck was successfully launched on May, 


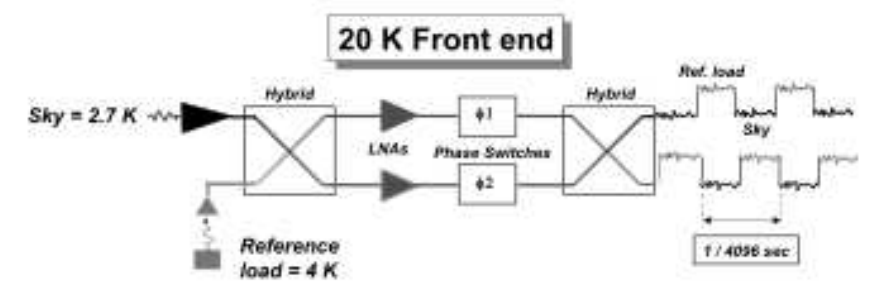

Fig. 1. Schematic view of a LFI Front-End Module (adapted from Mennella et al. (2009b)).

14, 2009 on an Ariane 5 rocket from Kourou spaceport in French Guiana.

The LFI is an array of 22 pseudo-correlation radiometers (Bersanelli et al. 2009), based on InP HEMT Low Noise Amplifiers (LNAs). Each radiometer is continuously comparing the signal from the sky with a stable reference signal provided by a unit, called $4 \mathrm{~K}$ Reference Load ( $4 \mathrm{KRL}$ ). It is composed of an assembly of small absorbing loads, thermally and mechanically connected to the HFI shield at a temperature of $\sim 4.5 \mathrm{~K}$. Each load is composed by a termination-like target, made of 1 ECCOSORB $^{\mathrm{TM}}$, facing a small pyramidal horn, connected to the $20 \mathrm{~K}$ radiometer reference arm.

The Planck instruments are cooled by a complex active system: a Hydrogen Sorption Cooler Bhandari et al. 2004; Morgante et al. 2009) keeps the LFI at $\sim 20 \mathrm{~K}$ and acts as pre-cooling stage for the HFI cryo-chain. This latter is composed by a mechanical 4K cooler (Bradshaw \& Orlowska 1997), which brings the HFI outer shield, where the 4KRL unit is mounted, at approximately $4.5 \mathrm{~K}$. Subsequent stages are composed a diluition cooler (internally pre-cooled at $1.6 \mathrm{~K}$ ), which brings the HFI bolometers to $\mathrm{T} \sim 100 \mathrm{mK}$ (Triqueneaux et al. 2006).

An overview of the $4 \mathrm{KRL}$ unit is presented in Section 2, in Section 3 we describe how the requirements for the 4KRL were derived; in Section 4 the mechanical, radiometric and thermal design of the 4KRL unit is reported; we describe in Section 5 the measured performance of the unit and we compare them with the requirements. Finally, some material properties are reported in the Appendix.

\section{An overview of the $4 \mathrm{~K}$ Reference Load Unit}

LFI uses a pseudo-correlation receiver design (Bersanelli et al. 2009). This radiometer concept is chosen to maximize the stability of the instrument by reducing the effect of non-white noise generated in the radiometer itself. In this scheme (see Figure 1), the difference between the inputs to each chains (the signal from the telescope and the signal from the $4 \mathrm{KRL}$ ) is continuously being observed. To minimize the $1 / \mathrm{f}$ noise of the radiometers, the reference blackbody temperature should be as close as possible to the sky temperature $(\sim 3 \mathrm{~K})$. To remove the effect of instability in the back-end amplifiers and detectors diodes, it is necessary to modulate the signals using phase switches. This design was chosen over a much simpler total-power scheme (consisting of one of the two parallel chains) because the latter exhibits inadequate gain stability at time scales larger than a few seconds (to be compared with the spin period, $60 \mathrm{~s}$ ).

The purpose of the $4 \mathrm{KRL}$ is to provide the radiometers with a low input offset (the radiometric temperature difference between the sky and the reference load). Reducing the input offset reduces the minimum achievable radiometer knee frequency for a given amplifier fluctuation spectrum (Seiffert et al. 2002). This minimum achievable knee frequency assumes perfect phase and gain matching in the two "legs" of the radiometer and assumes other ideal characteristics in radiometer components. An

\footnotetext{
${ }^{1}$ ECCOSORB is a TradeMark of Emerson and Cuming
}

ideal reference load temperature would match the sky temperature (approximately $2.7 \mathrm{~K}$ ), but there is no convenient spacecraft source of $2.7 \mathrm{~K}$ with sufficient cooling power. A gain modulation factor is introduced to compensate for this effect (Mennella et al. 2003). Moreover, minimising the input offset reduces the potential of multiplicative systematic effects to contaminate the measurement (Seiffert et al. 2002).

One of the main requirements for the $4 \mathrm{KRL}$ design was to minimize the heat load on the HFI to a value lower than 1 $\mathrm{mW}$. Safety considerations (a thermal short between the two instruments will prevent the HFI to work) lead to mechanically decouple the loads, mounted on the HFI external shield, from the LFI radiometers, at 20K. A system composed by an absorbing target (Reference Target - RT) at $4 \mathrm{~K}$ facing a receiving horn (Reference Horn - RH), connected to the radiometer at 20K, was selected (Figure 2).

\section{3. $4 \mathrm{KRL}$ requirements}

The design of the 4KRL unit is derived from performance required to the Low Frequency Instrument to obtain the Planck scientific objectives (Mandolesi et al. 2009; Bersanelli et al. 2009). We made a budget in which the overall requirements are split in the various contributions. We then set limits to each component to comply with the total requirement (both temperature stability and absolute temperature). The main quantities related to the $4 \mathrm{KRL}$ performance are the $1 / f$ knee frequency, which is linked to difference between the reference and sky temperature (Seiffert et al. 2002), the temperature stability (Mennella et al. 2002) and the 4KRL sensitivity to spurious RF components that could contaminate the reference signal. The requirements apply to the signal as measured at the input of the hybrid coupler in the FEM, where it is "mixed" with the signal from the sky. The design then has to be compliant with the allocated volume and mass for the unit and with thermo-mechanical constraints due to differential contraction when the reference load is cooled down to $4 \mathrm{~K}$. Moreover the design must be robust in term of safety, especially as far as vibrations at launch are concerned. The framework in which the 4KRL was developed is based on all these considerations.

The 4KRL RF design is intimately linked to its thermal design: the requirement on the maximum heat load on the HFI lead to a horn-target design, therefore allowing external signals to enter the radiometer reference arm (we call this effect leakage). This solution implies the presence of a gap in the radiometer reference arm, through which external spurious signals can leak in the radiometers. We must also consider the non-idealities of the reference system: mismatching between the load and the reference horn, losses in the reference arm. The need to dump the reference signal fluctuations imposed a trade-off on the thermal coupling between the reference targets and the heat sink, the HFI shields, impacting on the minimum temperature achivable by the loads.

We report here some useful definitions which are used in specifying the requirements. We express here the power in antenna temperature, $T_{A}$, in the Rayleigh Jeans approximation, and measured in Kelvin.

Ideally, the power that the radiometer receives (at the hybrid coupler) from a reference target at physical temperature $T_{R T}$ can be expressed as

$$
T_{\text {Load }}=\epsilon_{R T} T_{R T}
$$

where $\epsilon_{R T}$ is the emissivity of the reference target, and can be expressed, in the case of an ideal system, in terms of load reflectivity as $\epsilon_{R T}=\left(1-R_{R T}\right)$.

We identified the following terms contributing to the overall effective temperature:

$$
T_{4 K R L}=T_{\text {Load }}+T_{\text {leak }}+T_{\text {refl }}+T_{\text {loss }}
$$




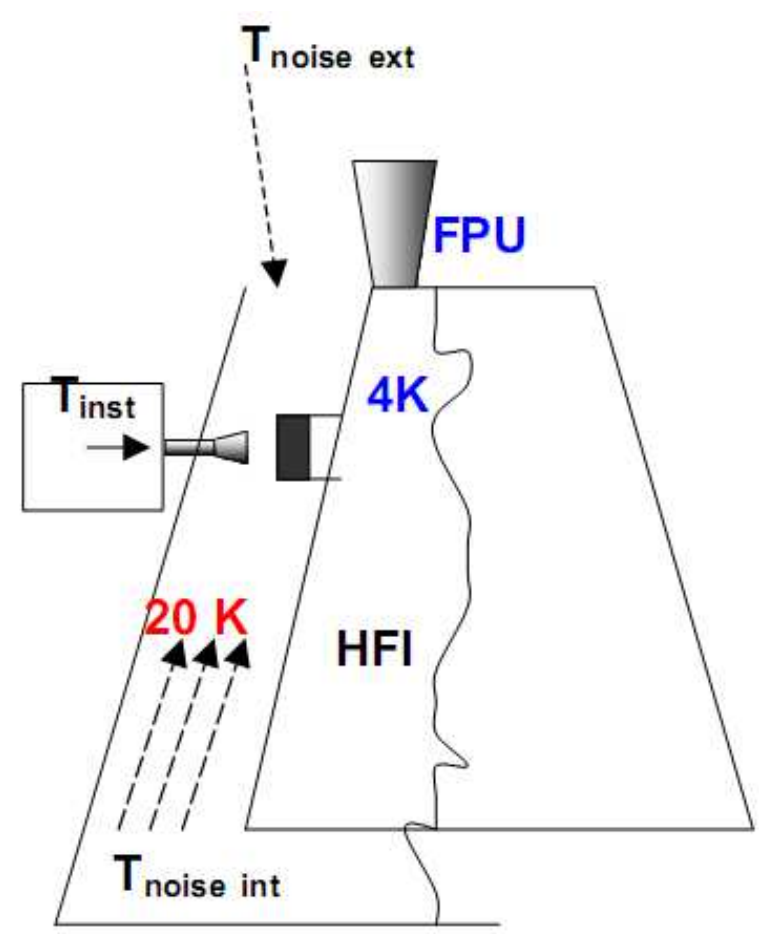

Fig. 2. Schematic representation signals reaching the 4KRL. One feed placed in the FPU looking at the sky, the $4 \mathrm{~K}$ HFI shield, one $4 \mathrm{KRL}$ (connected to the $4 \mathrm{~K}$ shield) and one $4 \mathrm{~K}$ reference horn (connected to the LFI main frame at $20 \mathrm{~K}$ ) are shown. On the left, a box representing the RF chain behind the horn is displayed.

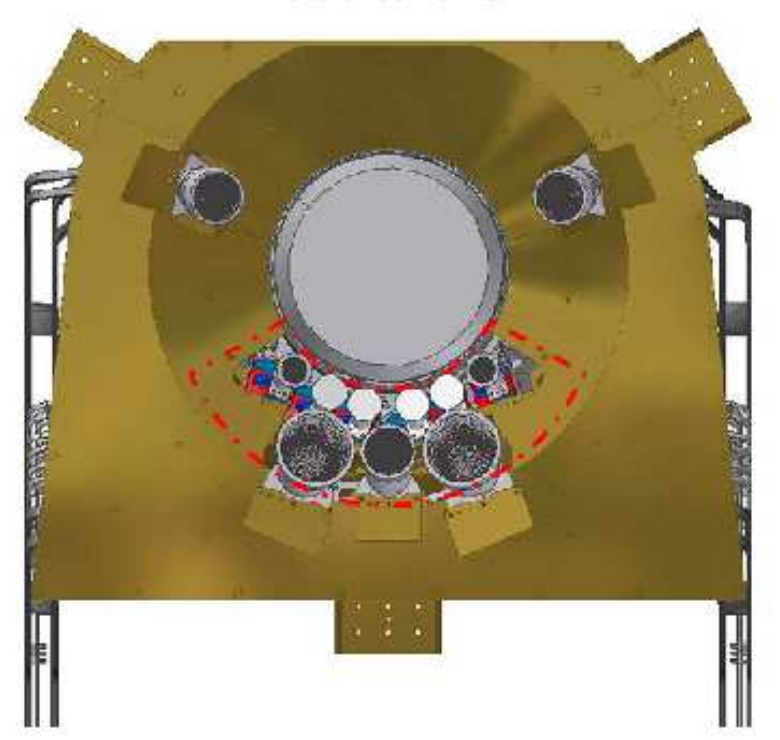

Fig. 3. Main frame top view: the red dot-dashed line highlights the gap around the feeds, allowing radiation from outside to enter the cavity

$$
\begin{aligned}
& \text { where } \\
& T_{\text {Load }}=T_{R T} \cdot\left(1-R_{R T}\right) \\
& T_{\text {leak }}=\left[T_{\text {int }} \cdot \epsilon_{\text {int }}+\right. \\
& \left.+\left(T_{\text {ext }}^{\text {sky }}+T^{\text {payload }} \cdot \epsilon_{\text {ext }}\right) \cdot S P O_{\text {ext }}\right] \cdot S P O_{\text {int }} \\
& T_{\text {refl }}=T_{N}^{\text {rad }} \cdot R_{R T} \\
& T_{\text {loss }}=T_{R H} \cdot L_{4 K R L}
\end{aligned}
$$

- $T_{R T}$ is the effective antenna temperature of the blackbody load, taking into account: HFI $4 \mathrm{~K}$ shield temperature at the interface of the reference loads and the thermal properties of the load and of the thermal link.

- $\left(1-R_{R T}\right)$ is the load emissivity, which include also the horn contribution.

- $T_{\text {leak }}$ is radiation leaking into the reference horn from the horn-target gap.

- $T_{\text {int }}$ is the temperature of the LFI-HFI cavity.

- SPO int,ext is the spillover dumping factor of external signals entering in the cavity (ext) and in the horn-target gap (int).

- $T_{r e f l}$ is the amplifier noise temperature, radiated in the radiometer reference arm and reflected back from targets.

$-T_{N}^{r a d}$ is the radiometer noise temperature.

- $T_{\text {loss }}$ is the contribution due to the ohmic loss of the reference horn (and the external reference waveguide for the 30 and $44 \mathrm{GHz})$.

- $R_{R T}$ is the mismatch at the horn aperture, when it is facing the target.

- $\epsilon_{\text {int, ext }}$ is the emissivity of the internal and the external environment, where the former refers to the cavity between the LFI and the HFI and the latter to the payload environment, inside the radiation shield surrounding the telescope.

- $W G$ : refers to the waveguide connecting each Reference Horn $(R H)$ to the radiometer reference arm.

It is evident that the load effective temperature, $T_{4 K R L}$ depends on target physical temperature and on the radiative behavior of the horn-target ensemble. We need the dominant contribution to be $T_{R T}$ and we need to set the requirements to design a unit which makes the other contribution negligible and well understood.

The critical areas are:

- The mismatching RL-RH, $R_{R T}$ : it causes power loss, and take the load away from having a perfect blackbody spectrum. It is strictly related to the reference horn geometry, load shape and material.

- The Spillover SPO: it causes a power loss from the load and power entrance from external (coming from instrument or sky). It depends on the RH near field pattern, on the intrinsic directional RL emissivity, on the RH-RL gap thickness.

- The power loss due to ohmic effects in the RH+WG unit. They attenuate the reference signal by $L_{4 K R L} \cdot T_{R T}$ and increase the antenna temperature by a larger quantity $L_{4 K R L}$. $T_{R H}$.

While the load absolute effective temperature is relevant for the $1 / f$ knee frequency, reference load signal fluctuations are by far more important, since they could mimic a sky signal.

Requirements on fluctuations are stringent: the 4KRL unit is designed to have spin synchronous signal (SS) fluctuations less than $1 \mu K$ per 30 arcmin sq. pixel on the final maps, after consolidated software removal techniques (Bersanelli et al. 2009).

Changes in the physical temperature of the reference load show up directly as errors in the output signal from the radiometer. The $4 \mathrm{KRL}$ signal stability requirement depends on the time scale of the variations. Variations on time scales long compared to the $60 \mathrm{~s}$ spin period are suppressed when the multiple observations of a given spot on the sky are combined; variations on time scales short compared to the radiometer sampling time are averaged out in a single sample. It is therefore necessary to distinguish three different cases: random, spin synchronous and sorption cooler synchronous temperature fluctuations. Each of the different contributors to temperature fluctuations will be analyzed considering these three cases. Random fluctuations are uncorrelated and, in general, add quadratically. SS fluctuations, in the worst case, add linearly.

If we differentiate Eq. 2. we can evaluate the different contributions: 


$$
\Delta T_{4 K R L}=\Delta T_{\text {Load }}+\Delta T_{\text {leak }}+\Delta T_{r e f l}+\Delta T_{\text {loss }}
$$

We assume the third term neglible, since the radiometer $T_{N}$ does not significantly fluctuate with time and we neglect also the last one, since it is balanced, to the first order, by the fluctuation on the radiometer sky arm. $\Delta T_{\text {Load }}$ is directly related to the HFI shield fluctuations, mainly induced by the Sorption Cooler (Bhandari et al. 2004; Morgante et al. 2009) and the 4K Cooler temperature oscillations (Lamarre et al. 2009). These can be damped by reducing the thermal link between the targets and the heat sink to an extent limited by mechanical contraints and by the maximum temperature allowed for the loads (i.e. the larger is the damping, the higher the load temperature).

Requirements on temperature oscillations of the HFI $4 \mathrm{~K}$ shield are different for different frequency intervals: the most stringent at low frequency (close to spin frequency). We therefore set complementary requirements on dumping factors between HFI interface to the $4 \mathrm{KRL}$ and the front surface of the load

$$
\Delta T_{R T}=D_{f} \cdot \Delta T_{H F I}
$$

The required values for $D_{f}$ are $<0.1$ and $<0.9$ at a period of $60 \mathrm{~s}$ and between $600 \mathrm{~s}$ and $1000 \mathrm{~s}$, respectively.

The term $\Delta T_{\text {leak }}$ is related to fluctuating signals entering the horn-target gap. They can be separated in two contributions: fluctuations of the cavity between the LFI and the HFI, where loads are located; fluctuations coming from telescope area and from the sky. These must be damped by assuming worst case values for $S P O_{i n t}$ and $S P O_{\text {ext }}$, respectively (Bersanelli et al. 2009).

Starting from top-level requirements, we derived requirements on each term contributing to equation 5.

The most critical SS signal at the LFI frequency is the CMB dipole, of the order of few mK (Fixsen et al. 1996): it must be dumped at a level below $\sim \mu \mathrm{K}$. This requires the total SPO factor to be $\leq-40 \mathrm{~dB}$. Since SS signals in the LFI-HFI cavity are expected, in the worst case assumption, lower than few $\mu \mathrm{K}$, we set:

$-S P O_{\text {int }} \leq-20 d B$

$-S P O_{\text {ext }} \leq-20 d B$

$T_{R T}$ should be known with an accuracy comparable to the knowledge of the CMB absolute temperature (Fixsen et al. 1996). Therefore we set:

$-R_{R T} \leq-20 d B$

In order to balance the two radiometer arms, the Insertion Loss $L_{4 K R L}$ in the reference arm needs to be of the same order of magnitude of the sky arm one. We then require:

$-L_{4 K R L} \leq 0.15 d B$

The requirement on the absolute temperature is $T_{4 K R L}<$ $5 K$, so to have a $1 / f$ knee frequency lower than $f_{k n e e}<$ $0.016 \mathrm{~Hz}$, corresponding to a spin period of 60 seconds. Moreover, the load need to operate over the whole radiometer bandwidth, $20 \%$ around each of the LFI frequencies (30, 44 and $70 \mathrm{GHz})$.

\section{Design and manufacturing of the $4 K R L$ unit}

While the first design constraint is the maximum thermal load the 4KRL may dissipate on the HFI, another relevant one is the location and the orientation of the LFI FEMs. They are placed along two almost concentric circles in the focal plane, each sky horn with a different orientation along its axis for polarization

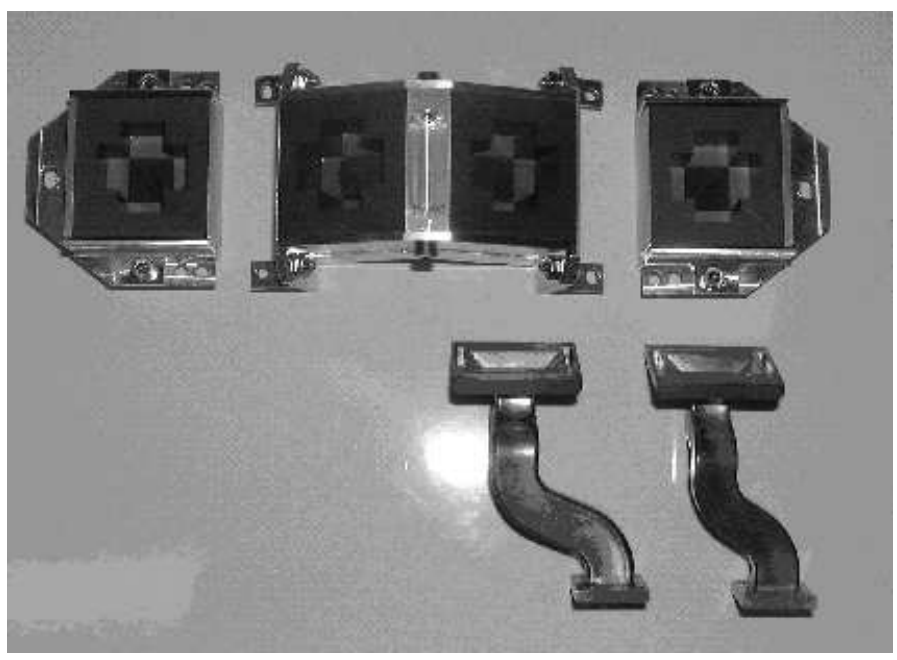

Fig. 4. 30GHz load. See Tables 1 and 2 for dimensions.

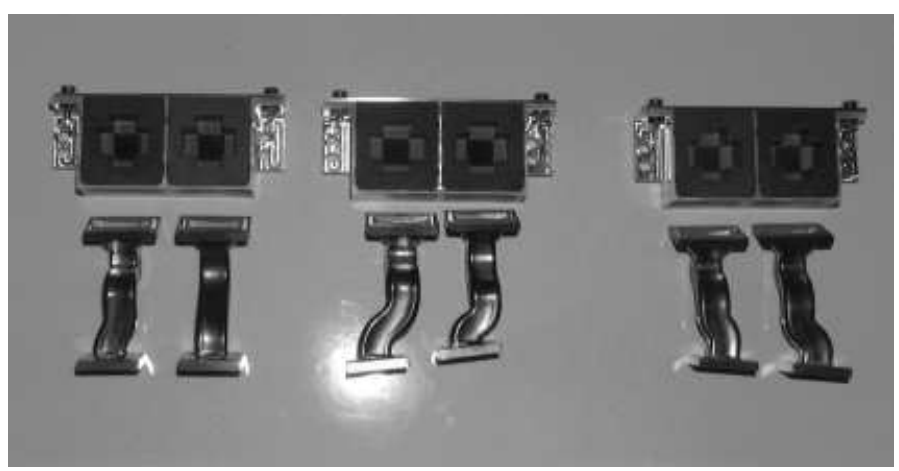

Fig. 5. 44GHz load. See Tables 1 and 2 for dimensions.

(see Sandri et al. (2009); Leahy et al. (2009); Bersanelli et al. $(2009))$. The 4KRL must also allow the integration of the HFI inside the LFI with all the loads already mounted.

Reference horns need to be small compared to the wavelength: this ruled out conical, corrugated horns, also because this design would have needed a transition from circular to rectangular waveguide, to match the apertures of hybrid. Target also need to be small and placed in the very near field of the reference horns to reduce the leak from the gap. The requirement to operate over the full radiometer bandwidth ruled out a resonant load, intrinsically narrow-band. The conceptual design is therefore based on small absorbing targets, mounted inside a metal enclosure ("case") to confine the radiation (see Figure 4 5). Their shape is optimized to reduce the reflectivity. Cases, supported by an Al structure, are mounted on the HFI using Stainless Steel thermal decouplers ("washers"), which allows to carefully control the thermal link behavior. The reference pyramidal horns are derived from waveguide flares and optimized coupled to the targets (see the $70 \mathrm{GHz}$ reference horn in Figure 6). The spacing between the horn and the targets is set to a nominal value of $1.5 \mathrm{~mm}$. Particular care is posed in minimizing the signal leaking through the horn-target gap.

\subsection{RF design}

The LFI is composed by 11 feed-horns, each one feeding two radiometers, one for each polarization (Bersanelli et al. 2009). The 4KRL unit is therefore composed by 22 reference loads (each one formed by a RT and a RH: 12, 6 and 4 for the 70, 44 and $30 \mathrm{GHz}$ LFI radiometers, respectively), assembled in 'twin' sub- 


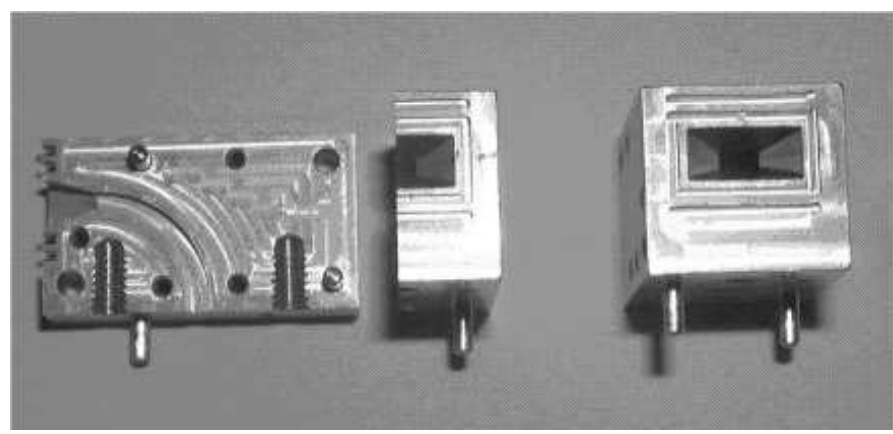

Fig. 6. $70 \mathrm{GHz}$ reference horn used for $4 \mathrm{KRL}$ testing. The waveguide connecting to the hybryd and grooves around the aperture are visible. Real horns are internal to FEMs (Varis et al. 2009). See Tables 1 and 2 for dimensions.

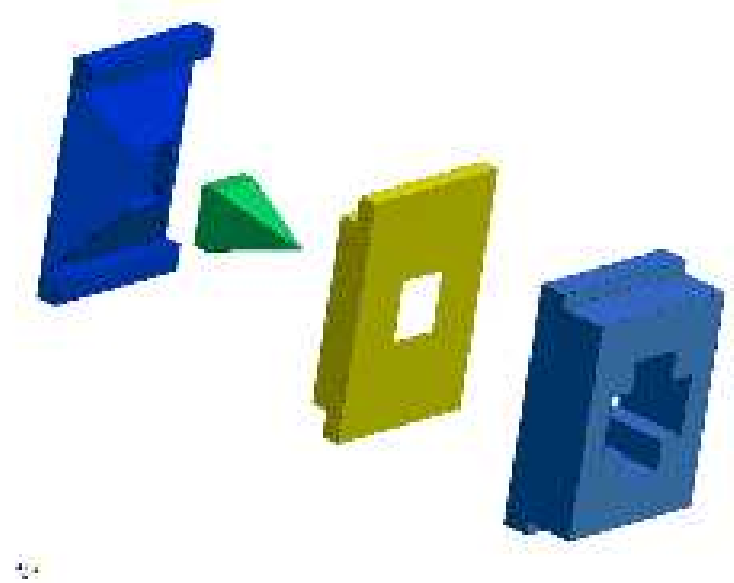

Fig. 7. Exploded view of a $70 \mathrm{GHz}$ target. The back part (base) is separated, in the $70 \mathrm{GHz}$ targets, in two sub-parts (blue and yellow). The pyramid (green) and the front part (cross, light blue) are visible.

units, shown in Figures 45 and 16 (70 GHz), one for each of the LFI Front-End Modules.

The development of a detailed RF design followed an iterative process: horns and targets were designed starting from analytical considerations; a first modellization using ModeMatching (MM) tools was applied to the reference horns; the Elegant BreadBoard (EBB) 4KRL model was built and the model results verified; a Finite Element Method modellization (FEM) was applied to the horn-target system, allowing to refine the design, which was verified in the calibration test on the Flight Model (FM) parts.

Mode Matching (MM) modelling: The first optimization on the horns (Cuttaia et al. (2004)) was devoted to minimize the mismatch at the aperture. Horn dimensions were scaled from 'standard' pyramidal horns, but it resulted in non standard electromagnetic design, where flare length is strongly reduced, impacting mainly the mismatch at the aperture and the antenna power pattern shape. The near-field pattern was calculated using GRASP[2: a typical example is reported in Figure 8

Each target is basically a rectangular ECCOSORB ${ }^{\mathrm{TM}} \mathrm{CR}$ block, shaped for optimal matching with the incoming field. The back part is made of highly absorbing CR117, while the front sector, made of CR110, reduces the mismatch. A cross-shaped void is grooved in the front part, to match the pyramidal horn

\footnotetext{
2 GRASP is a software developed by TICRA (Copenhagen,
}

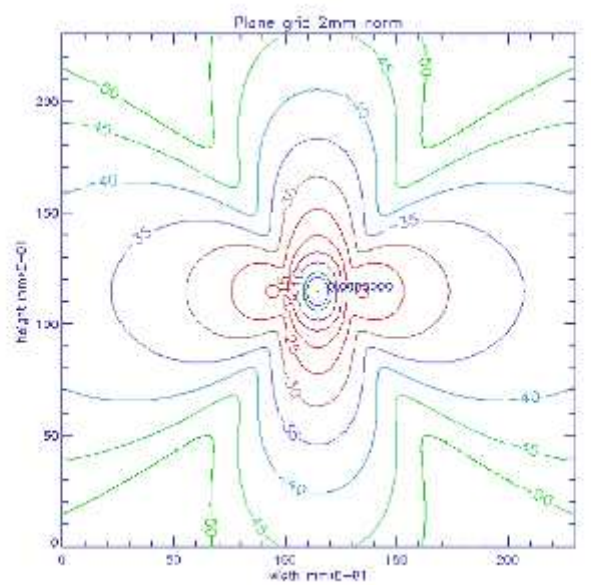

Fig. 8. Near-field contour plot of a 4 KRL pyramidal horn. The pattern is cross-shaped and targets are designed to match it.

near field. A pyramid, made of CR110, is placed in the center, contributing both to absorb the peak of the incoming field and to reduce reflections by geometrically tapering the absorber (see Figure 7). Other target designs (such as bed-of-nails) have been studied and some prototypes built and tested. Yet, the selected one showed the best performance for target of such small dimensions. At first, the design was linearly scaled with the frequency to all the LFI bands. We found that the return loss at the horn aperture is the dominant term. Some considerations were drawn: while horn aperture dimensions linearly scale with the frequency, waveguides dimensions and ECCOSORB ${ }^{\mathrm{TM}} \mathrm{RF}$ properties do not; for mechanical reasons, the horn-target distance is the same at all frequencies. Since MM modelling is not able to study the spillover at horn-target gap, a FEM modedelling technique (Cuttaia et al. 2004) was applied in the second optimizations step, where the horn-target combined system was separately optimized in each of the LFI bands.

FEM analysis required an adequate knowledge of electromagnetic characteristics: dedicated measurements have been performed on materials (see results in the Appendix).

The FEM conceptual scheme is reported in Figure 9, where ports are placed at the waveguides ends. TE10 mode is fed to the waveguides and it is possible to evaluate the S-parameters: the Sii parameter (Reflection) together with the Sij parameter (Cross Talk radiation coming from the port i detected from the port $\mathrm{j}$ when two coupled horns are modelled).

A radiation box, surrounded by perfectly matched layers, is placed around the system to evaluate the radiated field. In this way the spillover can be evaluated. Reference waveguides, with the actual complex routing, were separately modelled.

Finally, an accurate analysis was performed to investigate the stray-light contribution from sky external sources.

The final results of this long modelling process is a different design, both for horns (with waveguides) and loads, for each LFI band.

The final design for the horns, in addition to changes in the overall dimensions (flare angle and mouth size), is innovative in the introduction of rectangular $\lambda / 4$ grooves, around the aperture (Figures 6] and 19), able to significantly reduce the spillover (acting as rectangular waveguide chokes). Due to mechanical constraints, one complete groove and one on the long side (E-plane) are machined on each horn. $70 \mathrm{GHz} \mathrm{RH}$ have also been provided with a special small baffle, protruding $1.5 \mathrm{~mm}$ from Front End Modules, partially shielding the horn-target gap, therefore contributing in reducing the leaking radiation. 


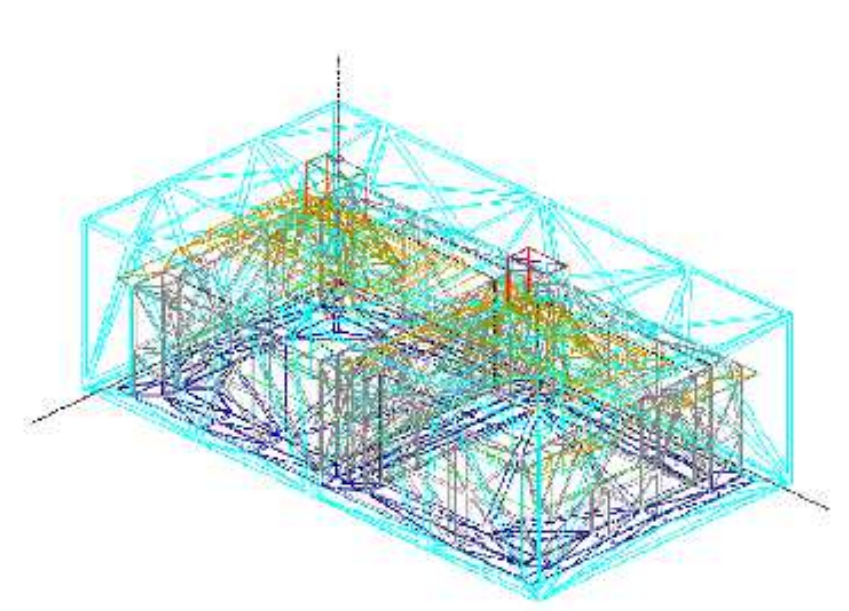

Fig. 9. Graphics representation of the FEM discretization in thetraedra: two horns and two targets are modelled together. The environment radiation box is represented in light blue
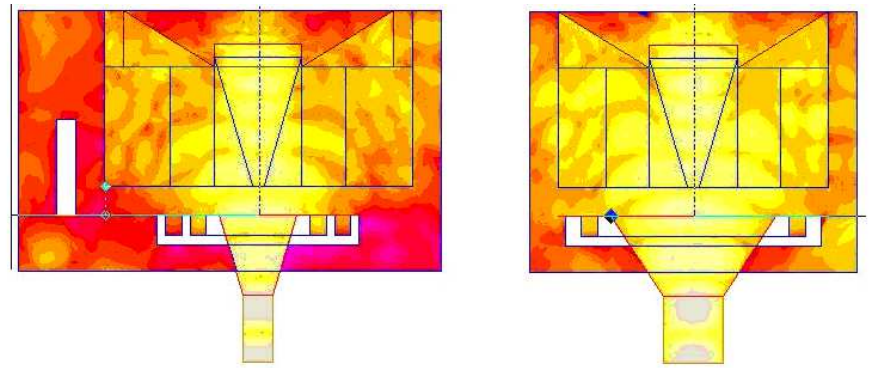

Fig. 10. E-field distribution for the $70 \mathrm{GHz}$ FM Model (left panel: $\mathrm{PHI}=90$ cut; right panel: $\mathrm{PHI}=0$ cut); lighter colours correspond to stronger E field. The white colour corresponds to areas (perfect conductors) where radiation does not enter. The metal case around the target is here replaced by a perfect conductor boundary condition. The metal baffle is evidenced (white) in the left panel.

The final target design for the 44 and $30 \mathrm{GHz}$ bands includes only small modification with respect to the original one: front dimensions in the E-field direction was increased and the thickness of the back CR117 layer was modified. Targets for the 70 $\mathrm{GHz}$ were deeply modified by FEM modelling results. The main differences are: the back ECR117 part is shaped as a pyramid, topped with a complementary part made of ECR110; the tip of the central pyramid is protruding $1 \mathrm{~mm}$ out of the front target surface. The final dimensions are reported in Table 1

\begin{tabular}{lccccccc}
\hline \hline Frequency & A1 & A2 & B & C & D & E & F \\
\hline GHz & & & $\mathrm{mm}$ & & & & \\
\hline 70 & 16.29 & 14.29 & 3.30 & 1.89 & 4.63 & 6.29 & 2.32 \\
44 & 24.73 & 22.73 & 5.00 & 3.00 & 7.36 & 10.00 & 3.69 \\
30 & 33.34 & 33.34 & 5.00 & 4.40 & 10.79 & 14.67 & 5.39 \\
\hline
\end{tabular}

Table 1. Reference Targets design dimensions. Refer to Figure 12 for the meaning of each column.

Moreover, mechanical contraints, imposed minor further modifications to targets, to avoid interference between parts and to allow their positioning in the final system. The impact of such

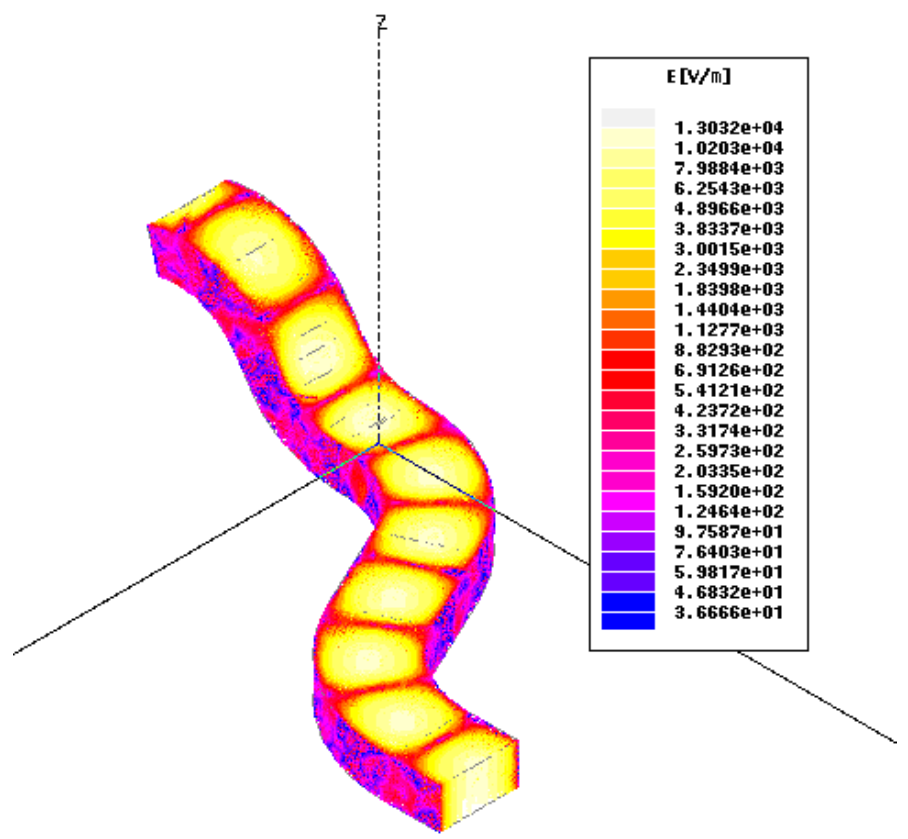

Fig. 11. Reference waveguide 28L modelled with FEM: the E-field distribution is represented with a logarithmic temperature colour palette)

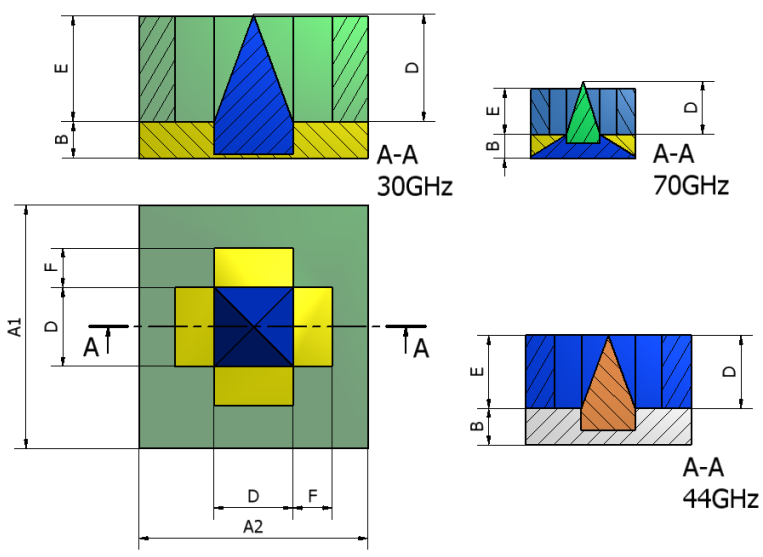

Fig. 12. Sketch of the reference target. Letters refer to Table 1. Note that the yellow part (base) is separated in two sub-parts at $70 \mathrm{GHz}$.

changes were measured during the characterization campaign and resulted to be negligible.

\subsection{Thermal design}

The 4KRL thermal design was studied to simplify as much as possible the interfaces. Thermal interface is dominated by con32 duction through thermal washers (the only point of contact be9 tween the RT and the HFI shield), allowing careful control of the heat transfer. Contact surfaces are mirror-polished at sub$\mu \mathrm{m}$ level. The method of bonding RT parts, which leave the base as the only thermal link, allows heat flow to be modelled in one dimension. Metal parts are assembled using Stainless Steel screws at high torque, to make thermal contact as close as possible to an ideal value.

Temperature of the $4 \mathrm{KRL}$ will be measured in flight by temperature sensors mounted inside the HFI shield. High sensitivity sensors are placed close to the 4KRL interface, both on the upper 


\begin{tabular}{lccccccccc}
\hline Frequency & WG & c & d & e & f & g & g1 & g2 & g3 \\
\hline GHz & \multicolumn{9}{c}{$\mathrm{deg}$} \\
\hline 70 & WR12 & 33.58 & 16.50 & 4.08 & 8.77 & 4.27 & 0.43 & 0.85 & 1.13 \\
44 & WR22 & 34.08 & 10.68 & 5.36 & 14.73 & 6.68 & 0.68 & 1.36 & 1.80 \\
30 & WR28 & 47.57 & 28.68 & 10.80 & 21.60 & 6.62 & 1.00 & 1.99 & 2.64 \\
\hline
\end{tabular}

Table 2. Reference Horns dimensions. Refer to Figure 13 for the meaning of each column.

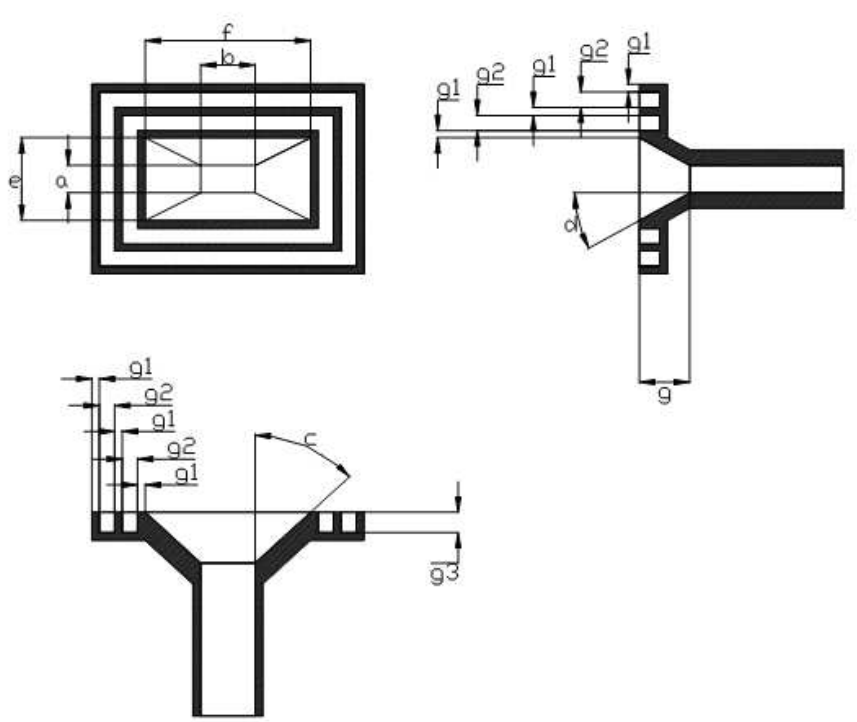

Fig. 13. Schematic view of a Reference Horn. Letters refer to Table 2

conical part (where $70 \mathrm{GHz}$ loads are) and on the lower, cylindrical one (30 and $44 \mathrm{GHz}$ loads). Sensitivity is of the order of $0.1 \mu \mathrm{K}$ and accuracy at the level of $\mathrm{mK}$ (Lamarre et al. 2009).

A thermal model of the $4 \mathrm{KRL}$ unit was realised. We started from the general design of the single reference target (see Figure 77). Each part of the target, the base, the cross and the pyramid is divided in five layers orthogonal to the pyramid axis. The aluminum case where the ECCOSORB ${ }^{\mathrm{TM}}$ parts are bonded and the support structure for the $70 \mathrm{GHz}$ loads are assumed as perfect thermal conductors, due to their small thickness and mass. The optimization of thermal washers allowed to increase the damping factor from the QM to the FM unit. The thermal model network representation is shown in fig. 14]

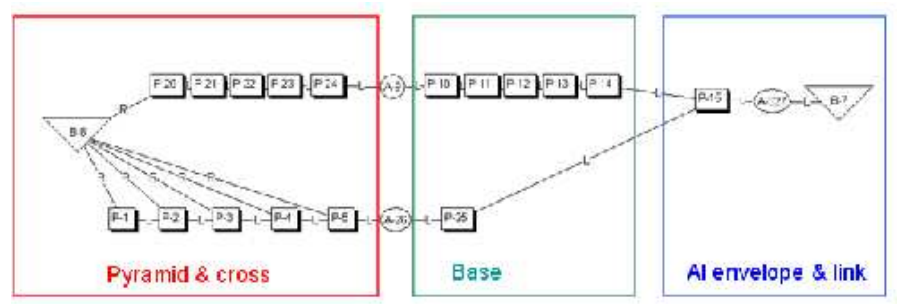

Fig. 14. The scheme of the thermal model for one of the $30 \mathrm{GHz}$ reference targets. The Aluminum envelope is condensed in one node linked to the HFI shield boundary node. The pyramid and the cross follow two parallel paths, coherently with heat flowing in the axial direction. The base of the pyramid is condensed in one node, while the base of the cross is split in five slices. Both pyramid and upper cross side are divided in five nodes each.
The main approximations of the current model are:

- due to the very small distance $(1.5 \mathrm{~mm})$ the radiative link is just considered with a perfect view factor, no analytic detailed radiative model was implemented

- Eccosorb CR110 thermal conductivity and specific heat are used for all ECCOSORB ${ }^{\mathrm{TM}}$ nodes

The second point above is evidenced when comparing, for instance, the transfer functions of thermal fluctuations with measured data. Figure [15] shows how the measured values suggests a higher cut frequency with respect to what is foreseen by the model. In any case, the agreement is satisfactory and it shows the level of model prediction of the loads dynamic behavior.

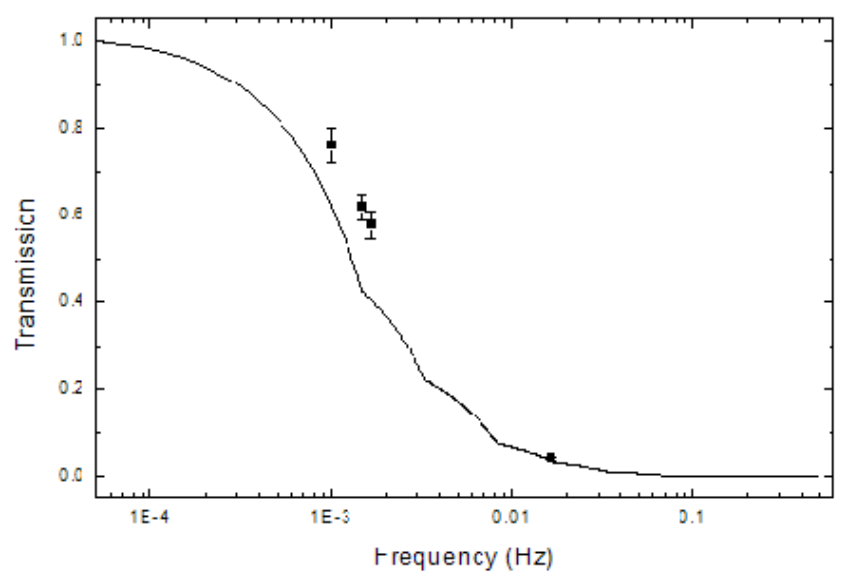

Fig. 15. Comparison between the simulated transfer functon foir a $44 \mathrm{GHz}$ reference target (line) and the measured data.

\subsection{Mechanical Design, Properties and Manufacturing}

The location of the LFI radiometers on the FPU (see Figure 3) lead to separate the loads in two different 'sectors': one for the $70 \mathrm{GHz}$ targets, forming the $4 \mathrm{KRL}$ upper sector, mounted on the cone supporting the HFI feed-horns; one for the 30 and $44 \mathrm{GHz}$ targets, the so-called $4 \mathrm{KRL}$ lower sector, mounted on the cylindrical part of the HFI $4 \mathrm{~K}$ shield. The $4 \mathrm{KRL}$ lower sector is then composed by the $30 \mathrm{GHz}$ target assembly (largest targets at the bottom of Figure 16) and three identical parts, one for each of the LFI $44 \mathrm{GHz}$ FEM, mounted at $120^{\circ}$ from each other (one of them is shown in Figure 16 over the $30 \mathrm{GHz}$ ones). Details in the mechanical design have been optimized to allow the integration of the unit on the HFI completely assembled. The final result is shown in Figure 16. While $\mathrm{RH}$ for the $70 \mathrm{GHz}$ radiometers were obtained inside the Front-End Modules (Figure 18), the location of the 30 and $44 \mathrm{GHz}$ FEMs required the introduction of external waveguides (Figure 19).

Each reference target is an assembly of 3 or 4 (at $70 \mathrm{GHz}$ ) parts obtained by casting ECCOSORB ${ }^{\mathrm{TM}}$. This material is an epoxy resin, loaded with iron particles with a typical dimension of few $\mu \mathrm{m}$ (see image in the Appendix). CR-110 differs from 


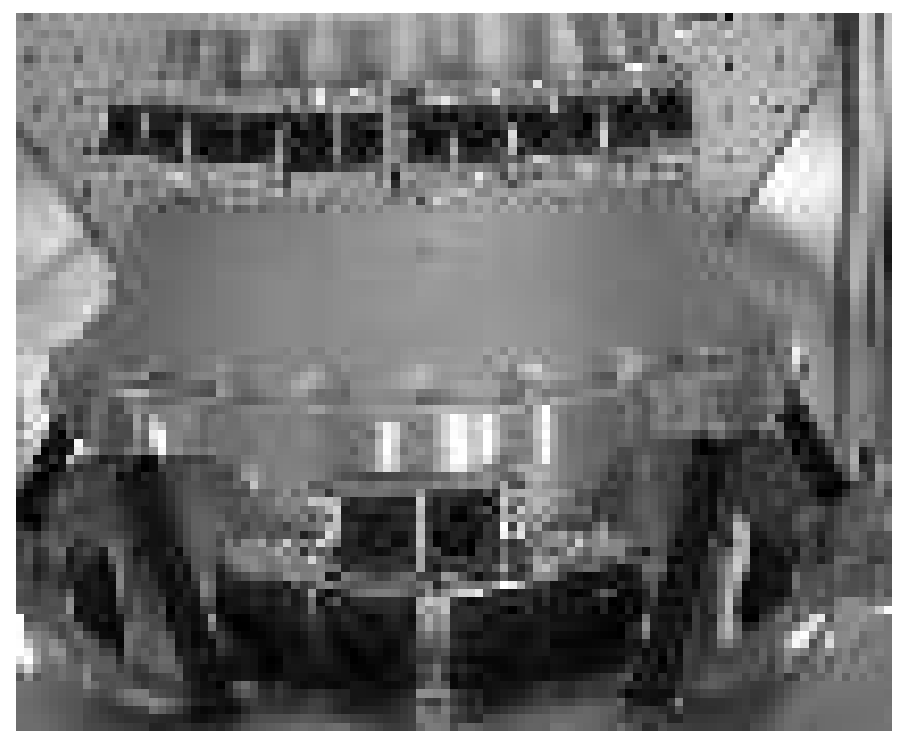

Fig. 16. The $4 \mathrm{~K}$ Reference Load unit on the HFI. The upper sector is mounted on the HFI cone. Note the reference target orientation, different for each 'twin' load. The $30 \mathrm{GHz}$ targets are shown in the lower sector. Reference horns are centered on each target, once the HFI is mated with the LFI. One of the $44 \mathrm{GHz}$ target is visible, while the other two are on the back side of the HFI $4 \mathrm{~K}$ shield, separated by $120^{\circ}$.

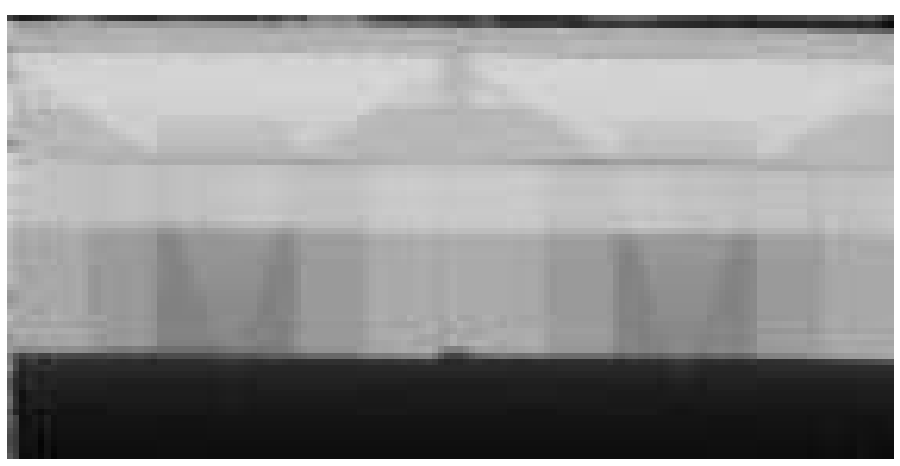

Fig. 17. X-ray image of one of the $70 \mathrm{GHz}$ reference target. Different ECCOSORB ${ }^{\mathrm{TM}}$ parts are clearly visible.

CR-117 in relative density of iron particles. The mould used was built in a flexible material (Derlin) in order to allow an easier removal operation and to avoid crack generation during the cooling process after the polymerization. Only the cross parts are machined to create the final shape. The required tolerance is achieved by an optimal control of the casting process, in particular for the thermal contraction derived from the polymerization temperature of $80 \mathrm{C}$ and from the high thermal contraction coefficient of the absorber. The process was studied to avoid the presence of air-bubbles inside the parts, a vacuum die-casting process was set to achieve this requirement. The curing time was enough fast to avoid iron particles to settle, avoiding the use of Cab-o-sil powder as in other applications (Hemmati et al. 1985; Mather et al. 1999). However, some parts were manufactured using the Cab-o-sil, and reflectance was measured. No significant difference was found. Some parts were also cut in order to verify the presence of bubbles and X-ray inspection was done in order to verify the quality of the process. A very careful check of the surface was done by using a microscope for all the components.
ECCOSORB $^{\mathrm{TM}}$ targets are assembled and then bonded, using Hysol EA9394 3 inside aluminum enclosures, made of certified Al6061-T6 to ensure optimal thermal properties at low temperature (see the Appendix for thermal data). Adhesive is not placed on target lateral surface, reducing mechanical stress induced by differential thermal expansion coefficients between ECCOSORB ${ }^{\mathrm{TM}}$ targets and $\mathrm{Al}$ cases. To increase safety, in the unfortunate case of a failure in the adhesive, ECCOSORB ${ }^{\mathrm{TM}}$ parts are also mechanically locked within the metal enclosure. Alignment pins ensure the correct alignment of the whole unit within $0.1 \mathrm{~mm}$.

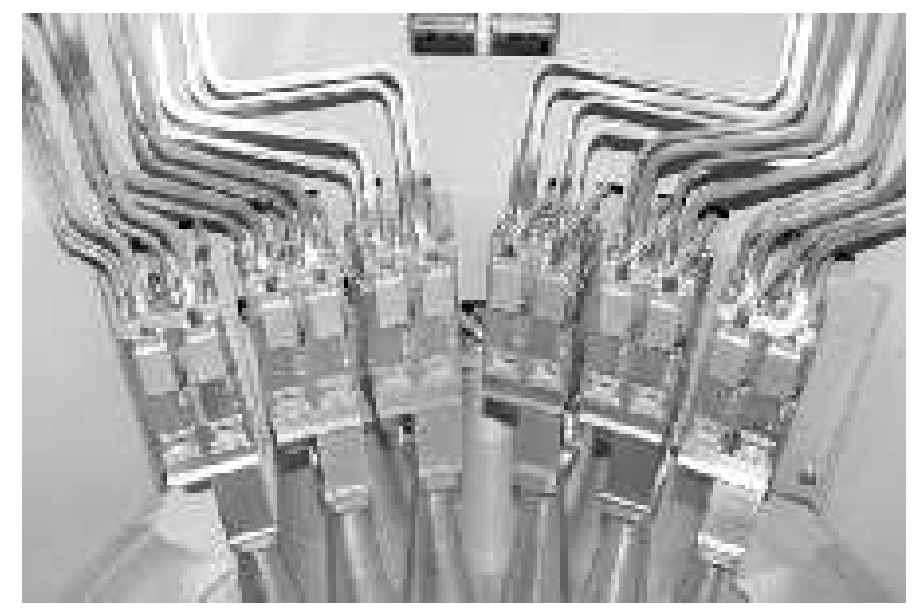

Fig. 18. $70 \mathrm{GHz}$ radiometers mounted on the LFI Main Frame structure. Reference Horns are clearly visible. Note also the step at the level of the OMT, used to cover the horn-target gap and reduce the spillover.

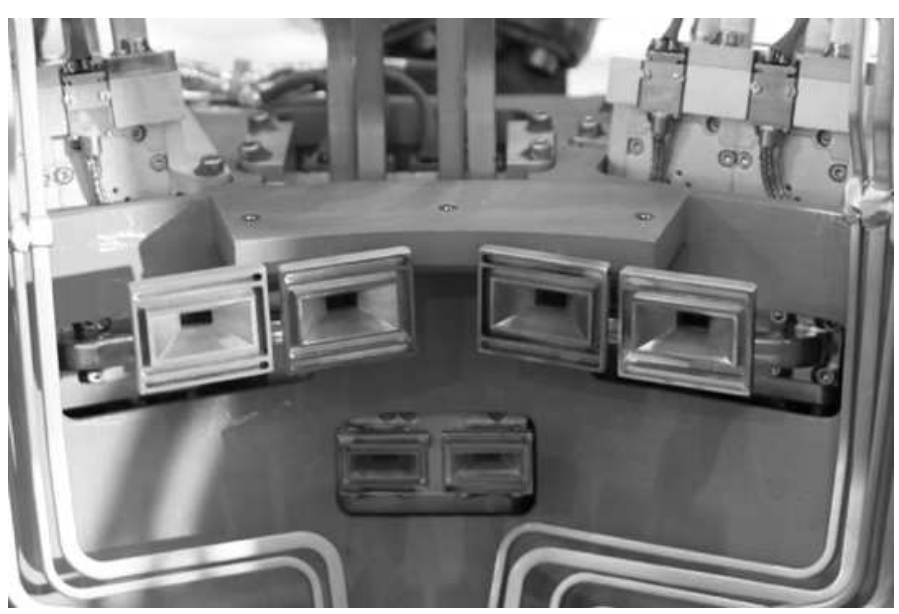

Fig. 19. $30 \mathrm{GHz}$ reference horns (upper, larger ones) and two of the $44 \mathrm{GHz}$ ones (lower) and their reference waveguides mounted on the LFI Main Frame structure. The grooves around the aperture are clearly visible.

Stainless steel (AISI304) thermal washers are interposed between the loads and the interface point to the HFI. These are small cylinders (typically $5 \mathrm{~mm}$ long, $1 \mathrm{~mm}$ wall thickness) whose dimension are optimized to dump temperature fluctuations in order to meet the requirements.

${ }^{3}$ Hysol is a Trade Mark of Loctite Aerospace. 
ECCOSORB $^{\mathrm{TM}}$ elastic module was measured on a representative sample, produced using the qualified process (see the Appendix). A FEM analysis was performed in order to verify the mechanical stress induced by the thermal contraction (see an example in Figure 201). It was confirmed that the most critical area is around the base of the target. In order to verify the capability of the reference target to tolerate the high vibrations of the launch, a FEM model of all the 4KRL was developed: the natural frequency was calculated and a random vibration analysis was performed on the modal model in order to verify the effect of the vibration test level on the structure (Figures 21 and 22).

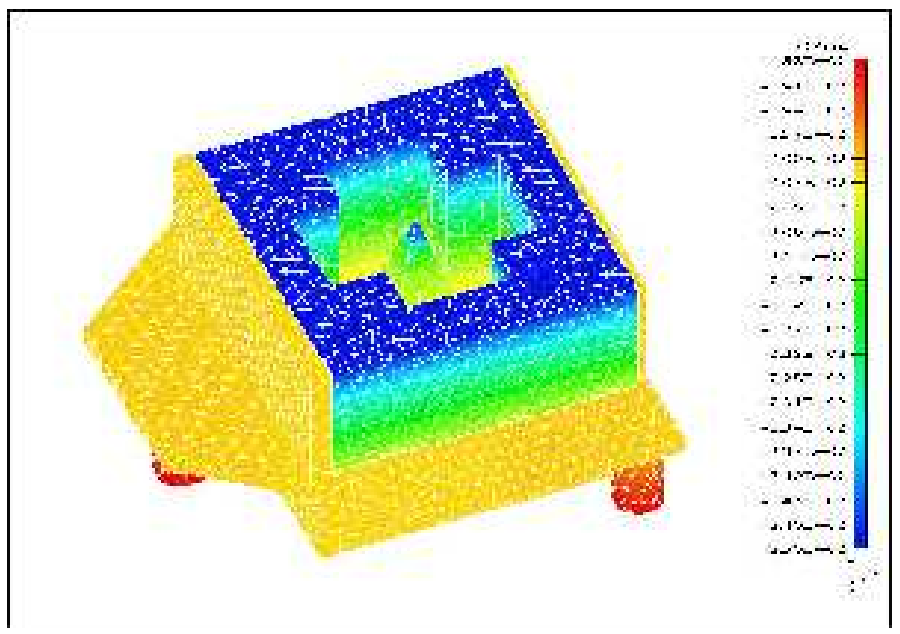

Fig. 20. FEM analysis results of relative temperature distribution on reference targets, used for thermo-structural analysis.

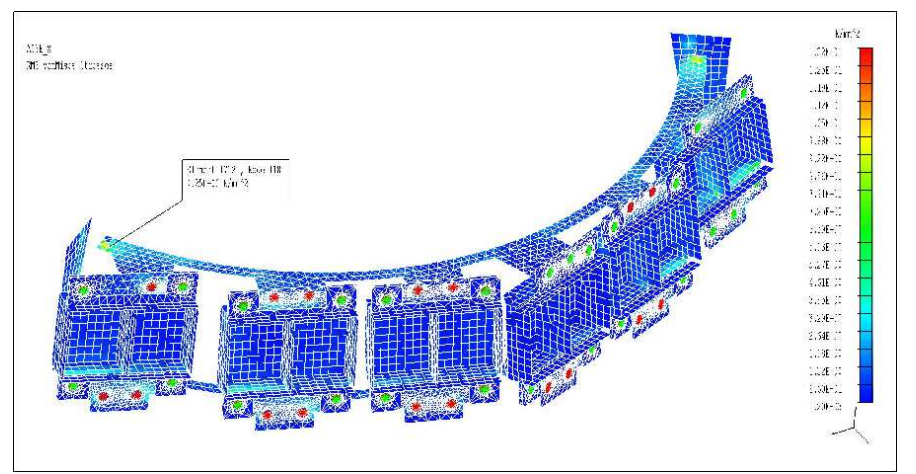

Fig. 21. Random vibration analysis of the $70 \mathrm{GHz}$ loads. The most critical areas, as expected, are the fixation points between the supporting structure and the lateral brackets.

Reference waveguides, used at 30 and $44 \mathrm{GHz}$, are characterized by a complex routing. Two or three bends are needed in a length less than $10 \mathrm{~cm}$. They are obtained by electro-forming pure copper on Al mandrel, which is then solved in caustic soda. In order to achieve the required dimensional tolerance, mandrels are electro-eroded, obtaining a final measured accuracy of about $0.05 \mathrm{~mm}$. Copper reference horns and gold-plated brass flanges, machined separately, are then soft-soldered. A small layer of gold is then flashed on the external surface to avoid oxidation. A first step in the qualification process was to determine the properties of the electroformed copper used for the waveguide, defining a test on specimens representative of a $30 \mathrm{GHz}$ waveguide (see data in the Appendix). Then, a FEM model of the waveguide was produced to determine the stress level and to determine the safety margin of the part.

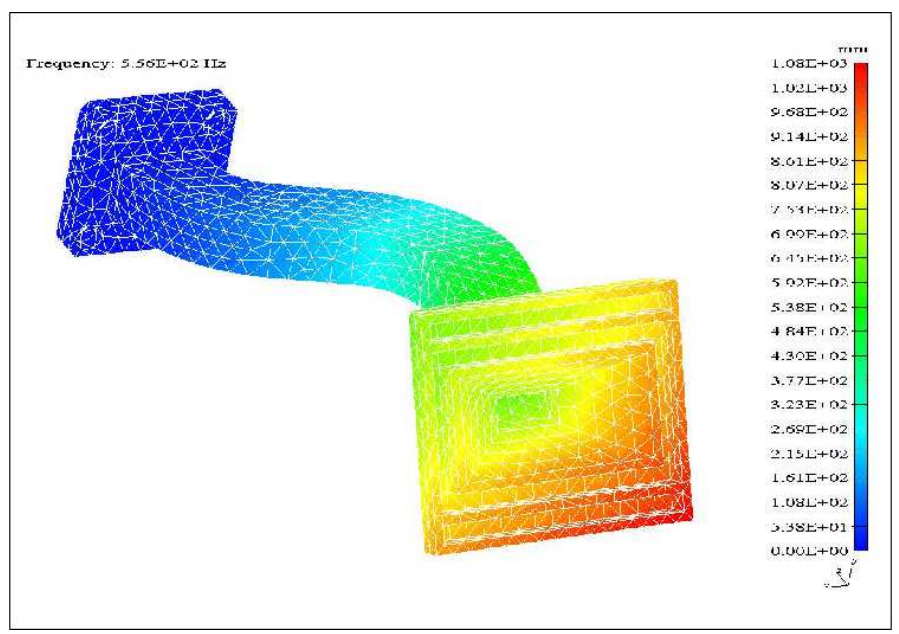

Fig. 22. Example of FEM analysis results on reference horns. It was used to determine the first natural frequency in terms of displacement.

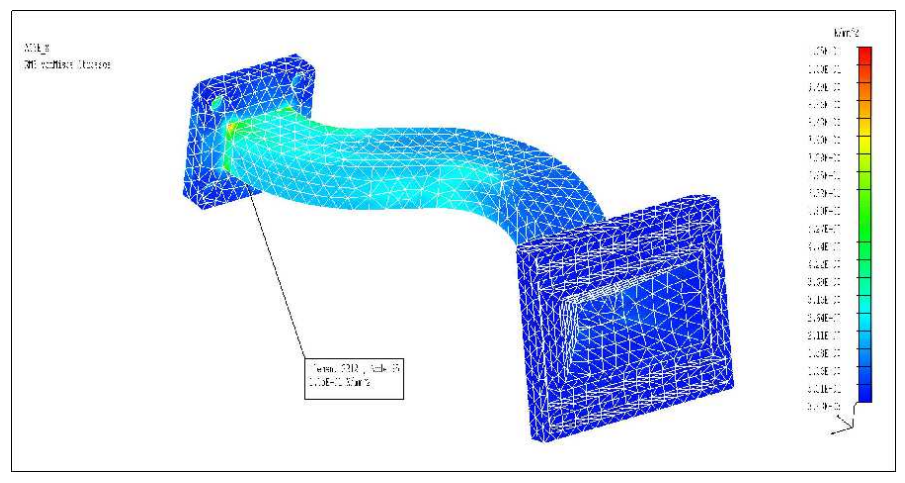

Fig. 23. Example of random vibration analysis on one of the $30 \mathrm{GHz}$ reference horns. The most critical area, as expected, is the interface between the waveguide and its flange.

The mass of the FM 4KRL unit is $572 \mathrm{~g}$ for the targets, including screws (mounted on the HFI) and $306 \mathrm{~g}$ for the waveguides (mounted on the LFI).

\section{Test activity}

The 4KRL unit model philosophy comprises four different models. Prototype Demonstrators (PD) and Elegant BreadBoard (EBB) models were used to design both horns and targets. Some loads were also used in testing the LFI radiometers prototypes. This activity allowed us to complete the 4KRL design. The Qualification Model (QM) was manufactured and submitted to a complete set of tests. Reference targets and horns were vibrated and successfully met the mechanical requirements. The $\mathrm{RF}$ and thermal test results were used to further refine the design (i.e. thermal dumping was increased, mounting structure was slightly modified to facilitate the integration), resulting in the Flight Model 4KRL design. The FM was then submitted to a complete characterization and acceptance test campaign, where $\mathrm{RF}$, thermal and mechanical properties were fully measured. 


\subsection{RF performance}

The 4KRL parts have been tested for RF performance before assembling into the instrument Focal Plane Unit (FPU). Each target was mounted on a 3 -axis positioning system, with micrometric control. A Scalar Network Analyser (SNA) was used to measure the RF performance, connected to the reference horns. While FM reference horns were used at 30 and $44 \mathrm{GHz}$, representative horn with adaptive flanges were adopted for the $70 \mathrm{GHz}$ loads, where the real horns are integrated into the radiometer Front-End Modules. Return Loss (RL), Insertion Loss (IL) and cross-talk between adiacent loads was measured for all targets. The results are reported in Tables 3 and Figures 24, 25] and 26.

\begin{tabular}{lccc} 
LFI RCA & Average IL (dB) & \multicolumn{2}{c}{ Average RL (dB) } \\
& RH & RH only & RH + RT \\
\hline \hline $18-\mathrm{M}$ & 0.16 & -20.99 & -20.14 \\
$18-\mathrm{S}$ & - & - & -20.18 \\
$19-\mathrm{M}$ & - & - & -20.54 \\
$19-\mathrm{S}$ & - & - & -20.25 \\
$20-\mathrm{M}$ & - & - & -20.18 \\
$20-\mathrm{S}$ & - & - & -19.56 \\
$21-\mathrm{M}$ & - & - & -20.58 \\
$21-\mathrm{S}$ & - & - & -20.48 \\
$22-\mathrm{M}$ & - & - & -20.07 \\
$22-\mathrm{S}$ & - & - & -20.29 \\
$23-\mathrm{M}$ & - & - & -19.57 \\
$23-\mathrm{S}$ & - & - & -19.83 \\
\hline $24-\mathrm{M}$ & 0.09 & -24.13 & -24.21 \\
$24-\mathrm{S}$ & 0.08 & -24.20 & -23.42 \\
$25-\mathrm{M}$ & 0.08 & -22.39 & -23.92 \\
$25-\mathrm{S}$ & 0.11 & -22.27 & -24.04 \\
$26-\mathrm{M}$ & 0.09 & -22.27 & -23.85 \\
$26-\mathrm{S}$ & 0.08 & -21.01 & -23.22 \\
\hline $27-\mathrm{M}$ & 0.11 & -26.59 & $-24.49{ }^{*}$ \\
$27-\mathrm{S}$ & 0.10 & -25.15 & $-26.45^{*}$ \\
$28-\mathrm{M}$ & 0.09 & -24.48 & -24.49 \\
$28-\mathrm{S}$ & 0.10 & -25.78 & -23.92 \\
\hline
\end{tabular}

Table 3. RF measured performance for the 4KRL: RH (+WaveGuide) Insertion Loss, RH (+WG) RL, RH (+WG) + RT Return Loss. $70 \mathrm{GHz}$ performance have been measured using a representative Reference Horn and waveguide, since RHs are internal to FEMs: this value is reported in the Table. RCAs 24-26 are the LFI $44 \mathrm{GHz}$ channels, 27 and 28 the $30 \mathrm{GHz}$ ones. $\mathrm{M}$ and $\mathrm{S}$ refer to Main and Side OMT arm, respectively. ${ }^{*}$ measured on the FM RTs and a representative Reference Horn.

We note a substantial uniformity of RL data at $70 \mathrm{GHz}$. Some of the loads, LFI 23 (M and S) and LFI $20-\mathrm{S}$ are close to the requirements of an average RL lower than $-20 \mathrm{~dB}$ at all the LFI frequencies. This is considered a minor effect, due to the specific geometry of those reference targets, and the parts have been accepted for flight. 30 and $44 \mathrm{GHz}$ loads show an overall RL well within the requirement. Minor differences in the measured values are present, since targets are not identical among each other: due to the orientation of the LFI FEMs, the shape some of the $70 \mathrm{GHz}$ target were adapted by cutting a small portion on the back of the ECCOSORB ${ }^{\mathrm{TM}}$ part; the central targets of the $30 \mathrm{GHz}$ assembly are also been adapted in the design, due to mechanical constraints; differences due to bonding can also be present. In Figure 26] we compare, as an example of the typical behavior, the results of reference horns matched to their targets (RH1+RT1, RH2+RT2) and to an ideal absorber (RH1, $\mathrm{RH} 2$ ). We observed that the RL performance is mainly dominated by the reference horn design. Measured performance is in good agreement with the FEM RF model. The effect of target-

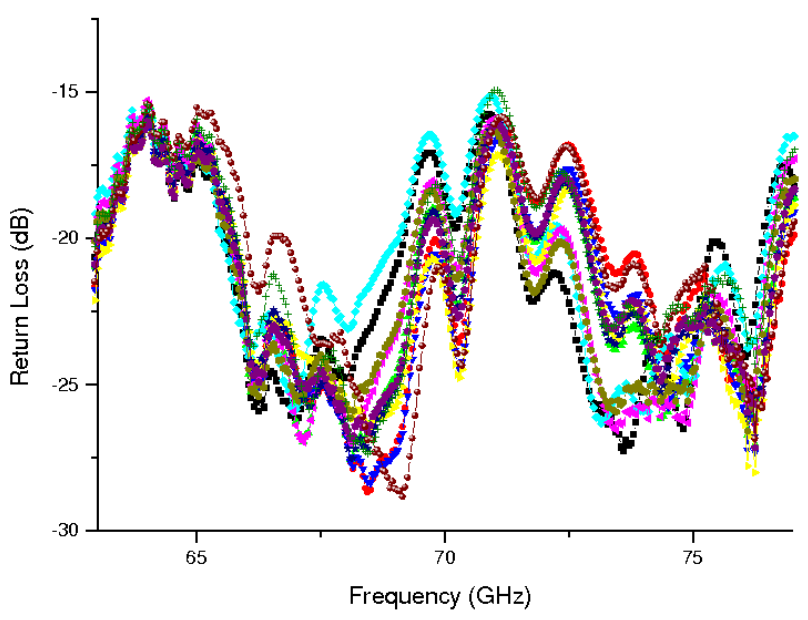

Fig. 24. Return Loss for the $70 \mathrm{GHz}$ targets coupled with a representative reference horn. To be noted the remarkable uniformity among all loads, due to a robust design and manufacturing.

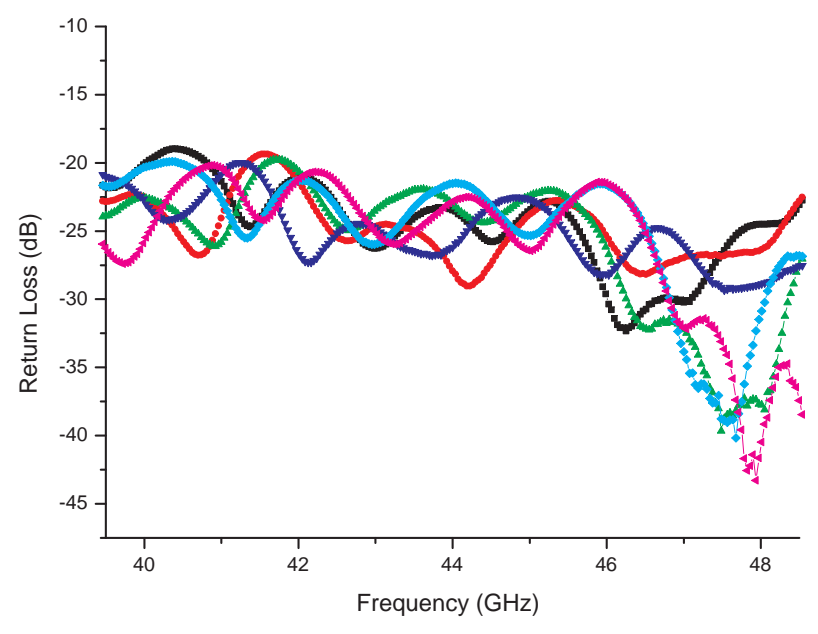

Fig. 25. Return Loss for the $44 \mathrm{GHz}$ targets coupled with their reference horns.

horn misalignment was also measured. The effect of $\pm 1 \mathrm{~mm}$ linear displacement on the three axis and of $\pm 1^{\circ}$ around them resulted in a maximum effect of $1 \mathrm{~dB}$ for all the LFI bands.

The $70 \mathrm{GHz}$ loads are the most affected by the spillover, because of their location close to the top of the Focal Plane Unit. To evaluate the SPO quantity, a dedicated detailed modelling was implemented, considering the whole cavity and the contributions from the external environment. It resulted in a gobal straylight rejection $\left(S P O_{e q}\right)$ better than $-60 \mathrm{~dB}$, well within the required limit.

The IL of the $4 \mathrm{KRL}$ waveguides and horns, measured on the 30 and $44 \mathrm{GHz}$ FM parts and averaged over the LFI bands, is reported in Figure 29] While the sensitivity of the SNA is sufficient to measure IL of the order of $0.1 \mathrm{~dB}$, the accuracy of the whole apparatus (mainly the directional coupler) leads to the large systematic uncertainty reported in the plot. The requirement of $I L_{4 K R L} \leq 0.15 \mathrm{~dB}$ is therefore considered met. Differences between the WGs are mainly due to differences in 


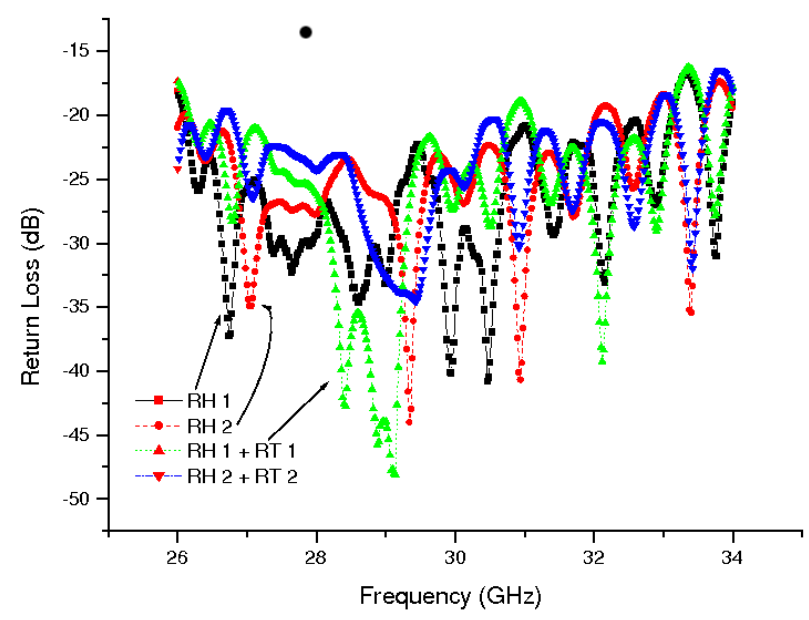

Fig. 26. $30 \mathrm{GHz}$ loads. The plots reports the RL measured for the horns in anechoic environment and coupled with their reference loads.

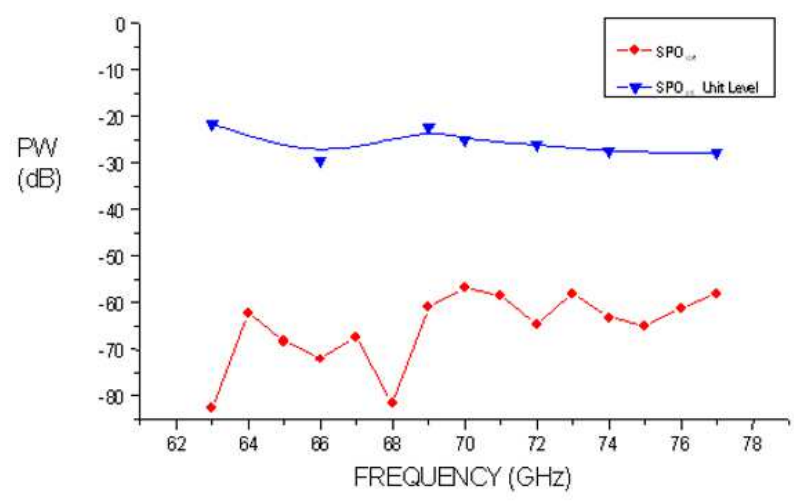

Fig. 27. Results of the spillover modelling for the $70 \mathrm{GHz}$ loads. Triangles refer to the internal spillover, diamonds to the global straylight rejection.

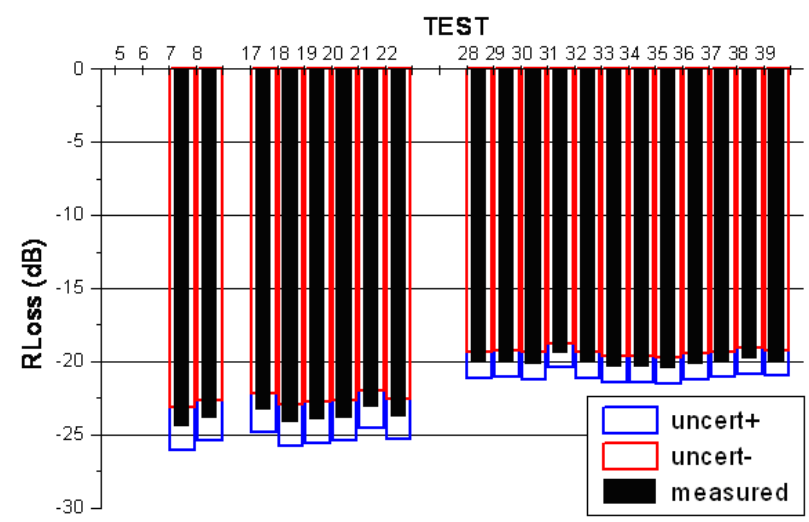

Fig. 28. Comparison of average RL measured on the LFI RTs coupled with their RHs. Tests $7-8$ refer to the $30 \mathrm{GHz}$ loads, tests $17-22$ to the $44 \mathrm{GHz}, 28-40$ to the $70 \mathrm{GHz}$ loads. Measurement systematic uncertainties, mainly due to the measurement setup, are also reported. The required values is $\mathrm{RL} \leq-20 \mathrm{~dB}$.

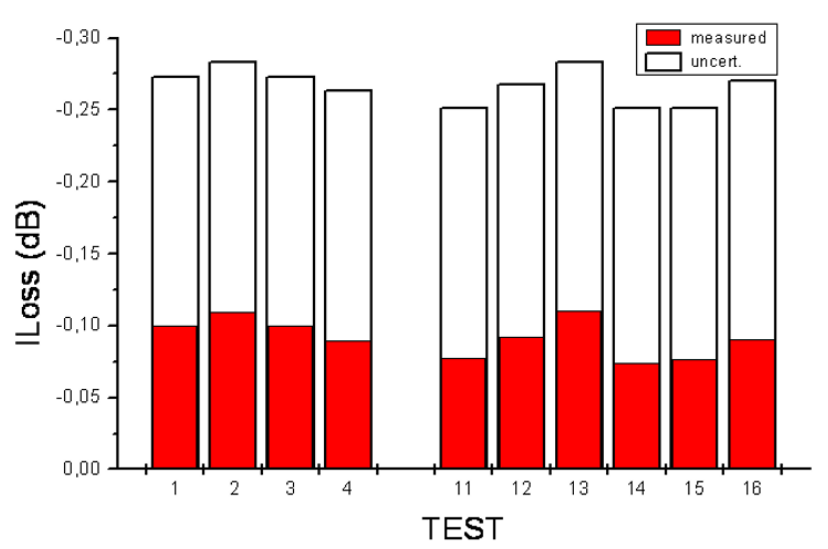

Fig. 29. Comparison of IL measured on the LFI reference wavegudes. Test $1-4$ refer to the $30 \mathrm{GHz}$ parts, tests $11-16$ to the $44 \mathrm{GHz}$ waveguides. Measurement systematic uncertainities are also reported.

routing and length. The $70 \mathrm{GHz}$ IL was modeled and compared with measure on the representative RH (see Figure 6), finding good agreement.

\subsection{Thermal performance}

Thermal tests were performed in the IASF-Bo $4 \mathrm{~K}$ cryo facility, equipped with a GM cooler, with an heat lift up to $1.5 \mathrm{~W}$ at $4 \mathrm{~K}$. The $4 \mathrm{KRL}$ targets were mounted on a Al6061-T6 support, with mechanical interfaces representative of the HFI one. A temperature controlled $\mathrm{Al}$ cylinder was located around the loads, simulating the radiative thermal interface at $20 \mathrm{~K}$. This setup simulated the real environment in the payload, where targets are mounted on the HFI $4 \mathrm{~K}$ shield in front of the quasicylindrical LFI main frame at about $20 \mathrm{~K}$. It was also used to test the susceptivity to fluctuations of the LFI.

Both the $\mathrm{Al}$ structures are connected to the cold flange via stainless steel thermal washers, whose dimensions are optimised to achive the desired stable temperatures with minimal heat load dissipated on the cooler cold stage. The thermal regulation is obtained with Minco film heaters fixed to the shields. Calibrated Lakeshore temperature sensors were used: silicon diode DT670, Cernox CX1050 and Germanium GR200A. Sensitivity and accuracy at $4 \mathrm{~K}$, using a LakeShore temperature controller 340 readout, is better than $1 \mathrm{mK}$ and $30 \mathrm{mK}$, respectively. The sensors are fixed with Aluminum tape on the face of the targets, as shown in Figure 30.

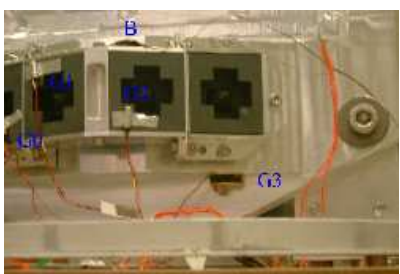

(a)

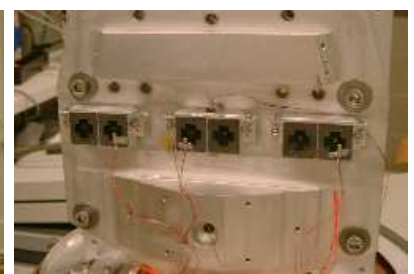

(b)
Fig. 30. Sensors mounting on the $30 \mathrm{GHz}$ (a) and $44 \mathrm{GHz}$ (b) reference load targets, during thermal tests. 
The steady state measurements were used to estimate the radiative heat load coming from the $20 \mathrm{~K}$ shield to the reference targets. The evaluation suffers of a large error because of the small temperature differences measured, comparable with the sensors accuracy. The global heat load is evaluated as (360 \pm 540) $\mu W$, compliant with the requirement.

The thermo-mechanical damping was evaluated from the transient test, inducing sinusoidal temperature fluctuation with periods of 60, 600, 667 (typical Sorption Cooler period) and 1000 seconds at the level of the attachment point of the loads on the support structures. The fluctuation at the level of the targets is then acquired (Figure 31) and the transfer function (amplitude and phase) are estimated by the ratio of the amplitudes. The final results are summarized in the Table 4 .

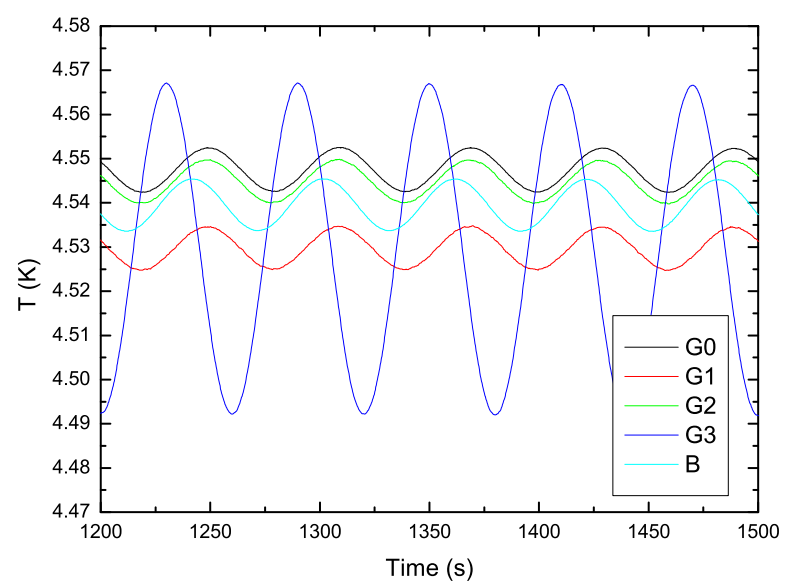

Fig. 31. The temperature fluctuation damping measured during the $44 \mathrm{GHz}$ thermal test. The $60 \mathrm{~s}$ fluctuation test data are shown. The blue line $(\mathrm{B})$ is the temperature curve of the source of fluctuations while the damped curves refer to the aluminum case (G3) and target faces (G0-G2).

The damping factor for the $44 \mathrm{GHz}$ and $70 \mathrm{GHz}$ loads with 60 s period fluctuation was found slightly above the requirements. The effect was projected on simulated CMB maps, and it was demonstrated that the final effect could be safely removed by destriping techniques (Maino et al. 2002).

A set of additional functional tests consisting in thermal cycles of the load were performed to check the survival to thermomechanical stress.

\section{Conclusions}

The complete $4 \mathrm{KRL}$ unit was used for the LFI test activity at instrument level, where the LFI radiometer performance were measured in cryo environment. The unit was mounted on a dedicated support, thermally stabilized at a temperature around $20 \mathrm{~K}$, as well as the sky load. Performance of the LFI are then extrapolated at operating temperature. No temperature sensor was placed on the targets to measure the damping factor, but the global results on the 1 /f noise performance was satisfying the performance of the LFI. The effect of load temperature variation was also visible in the radiometer output and it was possible to remove it in data analysis. These results are fully described in (Mennella et al. 2009a).

After this activity, the $4 \mathrm{KRL}$ unit was integrated on the HFI in October 2006. The final location was measured with respect to the design, using a 3-D measurement arm. The final alignment was found within a fraction of a millimeter with respect to the design values. After the integration of the HFI into the LFI, the clearance between reference horns and targets was verified using an endoscope.

Once the unit is integrated on the payload, thermal stability of the RTs is directly linked to the HFI $4 \mathrm{~K}$ cooler performance, while temperature data relevant to the $4 \mathrm{KRL}$ unit are provided by HFI sensors. These are mounted inside the HFI $4 \mathrm{~K}$ shield and the temperature of the RTs is extrapolated using the thermal model described in a previous section. The $70 \mathrm{GHz}$ loads, being located close to the HFI 4K flange, where the HFI sky horns, are mounted, will take advantage of the thermal stabilization obtained by a PID controller on the HFI internal shield. The 30 and $44 \mathrm{GHz}$ loads, being located farther from the controlled stage, will be more influenced by the HFI $4 \mathrm{~K}$ stages fluctuations. A full description of the HFI cryo chain and its behavior can be found in Lamarre et al. (2009).

Preliminary results from ground based test at satellite level, performed at the Centre Spatiale de Lige (CSL), confirmed the excellent noise properties of the LFI radiometers, especially for those concerning the $1 / \mathrm{f}$ knee frequency, related to the $4 \mathrm{KRL}$ performance. Therefore, indirectly, the correct behavior of the 4KRL unit was assessed (see Mennella et al. (2009a) for details).

We have presented in this paper the design, development, manufacturing and test activities of the $4 \mathrm{~K}$ Reference Load Unit for the Low Frequency Instrument on-board the Planck satellite. This work allowed to build an innovative, very compact, high performance cryogenic calibrator for millimeter wave radiometers, compliant with very stringent requirements. Relevant material data were collected from the literature and directly measured, where required.

Acknowledgements. Planck is a project of the European Space Agency with instruments funded by ESA member states, and with special contributions from Denmark and NASA (USA). The PlanckLFI project is developed by an International Consortium lead by Italy and involving Canada, Finland, Germany, Norway, Spain, Switzerland, UK, USA. The Italian contribution to Planck is supported by the Italian Space Agency (ASI). The US Planck Project is supported by the NASA Science Mission Directorate. We would like to thank ESA material division for the support given during test on materials. G. Dall'Oglio, L. Pizzo, Todd Gaier and Mike Seiffert helped us in the early stages of the 4KRL development.

\section{References}

Bersanelli, M., Mandolesi, N., Butler, R., et al. 2009, A\&A, submitted Bhandari, P., Prina, M., Bowman, R. C., et al. 2004, Cryogenics, 44, 395

Bradshaw, T. W. \& Orlowska, A. H. 1997, in ESA Special Publication, Vol. 400, Sixth European Symposium on Space Environmental Control Systems, ed. T.-D. Guyenne, 465-+

Cuttaia, F., Valenziano, L., Bersanelli, M., et al. 2004, Nuclear Instruments and Methods in Physics Research A, 520, 396

Fixsen, D. J., Cheng, E. S., Gales, J. M., et al. 1996, ApJ, 473, 576

Halpern, M., Gush, H. P., Wishnow, E., \& de Cosmo, V. 1986, Appl. Opt., 25, 565

Hemmati, H., Mather, J. C., \& Eichhorn, W. L. 1985, Appl. Opt., 24, 4489

Lamarre, J. M., Puget, J. L., Bouchet, F., et al. 2003a, New Astronomy Review, 47, 1017

Lamarre, J. M., Puget, J.-L., Bouchet, F., et al. 2009, A\&A

Lamarre, J.-M., Puget, J. L., Piat, M., et al. 2003b, in Presented at the Society of Photo-Optical Instrumentation Engineers (SPIE) Conference, Vol. 4850, IR Space Telescopes and Instruments. Edited by John C. Mather . Proceedings of the SPIE, Volume 4850, pp. 730-739 (2003)., ed. J. C. Mather, 730-739

Leahy, J., Bersanelli, M., D'Arcangelo, O., et al. 2009, A\&A, Submitted

Maino, D., Burigana, C., Górski, K. M., Mandolesi, N., \& Bersanelli, M. 2002, A\&A, 387, 356

Mandolesi, N., Bersanelli, M., Burigana, C., et al. 2000, A\&AS, 145, 323

Mandolesi, N., Bersanelli, M., Butler, R., et al. 2009, A\&A, Submitted 


\begin{tabular}{lcccc}
\hline Freq. & $\mathrm{D}(60 \mathrm{~s})$ & $\mathrm{D}(600 \mathrm{~s})$ & $\mathrm{D}(667 \mathrm{~s})$ & $\mathrm{D}(1000 \mathrm{~s})$ \\
\hline $30 \mathrm{GHz}$ & $0.080 \pm 0.004$ & $0.60 \pm 0.03$ & $0.64 \pm 0.03$ & $0.78 \pm 0.04$ \\
$44 \mathrm{GHz}$ & $0.133 \pm 0.007$ & $0.81 \pm 0.04$ & $0.85 \pm 0.04$ & $0.91 \pm 0.05$ \\
$70 \mathrm{GHz}$ & $0.131 \pm 0.007$ & $0.72 \pm 0.04$ & $0.75 \pm 0.04$ & $0.85 \pm 0.04$ \\
\hline
\end{tabular}

Table 4. Thermal fluctuation damping measured for the reference loads at different frequencies

Mandolesi, N., Bersanelli, M., Butler, R. C., et al. 2002a, in American Institute of Physics Conference Series, Vol. 616, Experimental Cosmology at Millimetre Wavelengths, ed. M. de Petris \& M. Gervasi, 193-201

Mandolesi, N., Villa, F., \& Valenziano, L. 2002b, Advances in Space Research, 30, 2123

Mather, J. C., Fixsen, D. J., Shafer, R. A., Mosier, C., \& Wilkinson, D. T. 1999, ApJ, 512, 511

Mennella, A., Bersanelli, M., Aja, B., et al. 2009a, A\&A, Submitted

Mennella, A., Bersanelli, M., Burigana, C., et al. 2002, in American Institute of Physics Conference Series, Vol. 616, Experimental Cosmology at Millimetre Wavelengths, ed. M. de Petris \& M. Gervasi, 229-233

Mennella, A., Bersanelli, M., Cappellini, B., et al. 2004, in American Institute of Physics Conference Series, Vol. 703, Plasmas in the Laboratory and in the Universe: New Insights and New Challenges, ed. G. Bertin, D. Farina, \& R. Pozzoli, 401-404

Mennella, A., Bersanelli, M., Seiffert, M., et al. 2003, A\&A, 410, 1089

Mennella, A., Villa, F., Terenzi, L., et al. 2009b, J-INST, This issue

Morgante, G., Pearson, D., Stassi, P., et al. 2009, J-INST, This issue

Papendrecht, G. 2002a, Micro-VCM outgassing test on Hysol EA9361 and ECCOSORB CR110, Tech. Rep. TOS-QMC Report 2002/145, ESA Material Division

Papendrecht, G. 2002b, Micro-VCM outgassing test on two adhesives, Tech. Rep. TOS-QMC Report 2002/80, ESA Material Division

Peterson, J. B. \& Richards, P. L. 1984, International Journal of Infrared and Millimeter Waves, 5, 1507

Puget, J.-L. 2004, in COSPAR, Plenary Meeting, Vol. 35, 35th COSPAR Scientific Assembly, 4559

Sandri, M., Bersanelli, M., Burigana, C., et al. 2004, Memorie della Societa Astronomica Italiana Supplement, 5, 411

Sandri, M., Bersanelli, M., Mennella, A., et al. 2009, A\&A, Submitted

Seiffert, M., Mennella, A., Burigana, C., et al. 2002, A\&A, 391, 1185

Tauber, J., Norgaard-Nielsen, H. U., Amiri Parian, J., et al. 2009, A\&A

Tauber, J. A. 2001, in IAU Symposium, Vol. 204, The Extragalactic Infrared Background and its Cosmological Implications, ed. M. Harwit, 493

Tauber, J. A. 2005, in IAU Symposium, Vol. 201, New Cosmological Data and the Values of the Fundamental Parameters, ed. A. N. Lasenby \& A. Wilkinson, 86

Tauber, J. A. 2006, in The Many Scales in the Universe: JENAM 2004 Astrophysics Reviews, ed. J. C. Del Toro Iniesta, E. J. Alfaro, J. G. Gorgas, E. Salvador-Sole, \& H. Butcher, 35

Triqueneaux, S., Sentis, L., Camus, P., Benoit, A., \& Guyot, G. 2006 , Cryogenics, 46, 288

Valenziano, L., Sandri, M., Morgante, G., et al. 2007, New Astronomy Review, 51, 287

Varis, J., Hughes, N., Laaninen, M., et al. 2009, J-INST, This issue

Villa, F., Mandolesi, N., Bersanelli, M., et al. 2002, in American Institute of Physics Conference Series, Vol. 609, Astrophysical Polarized Backgrounds, ed. S. Cecchini, S. Cortiglioni, R. Sault, \& C. Sbarra, 144-149

\section{Appendix A: Material data}

The 4KRL targets are made of ECCOSORB ${ }^{\mathrm{TM}} \mathrm{CR}$-series. This absorbing material, widely used in microwave passive components, has already been used in space applications and at low temperature (Peterson \& Richards 1984; Mather et al. 1999). However, electrical and thermal properties are not well documented and it has been necessary to qualify it for our application. RF, thermal, mechanical and outgassing properties of Eccosorb CR-110 and CR-117 were measured at room temperature. RF transmittance and reflectivity data are reported in Figures A.1 and A.2

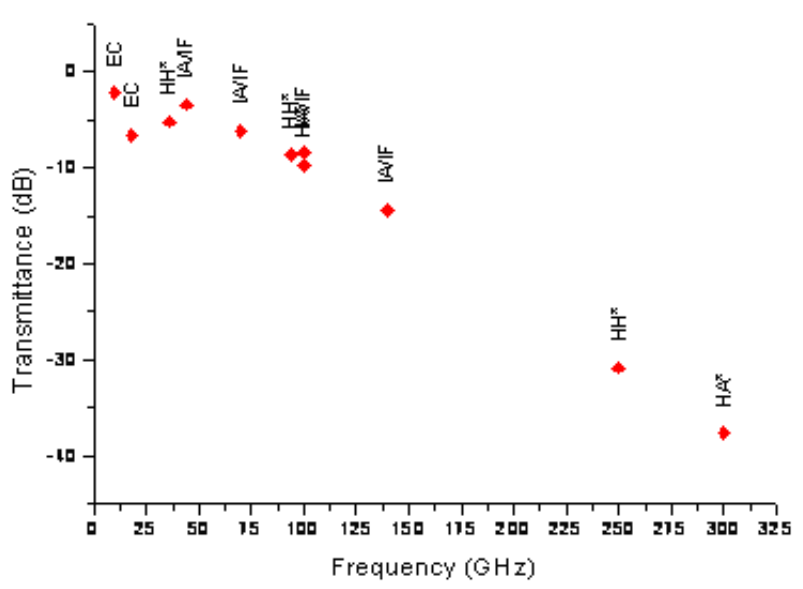

Fig. A.1. Logarithmic transmittance (expressed in dB) for ECCOSORB CR110. ECR: Emerson and Cumings, $\mathrm{HH}$ Hemmati et al. (1985), Ha: Halpern et al. (1986), $\mathrm{IA} / \mathrm{IF}$ : this work

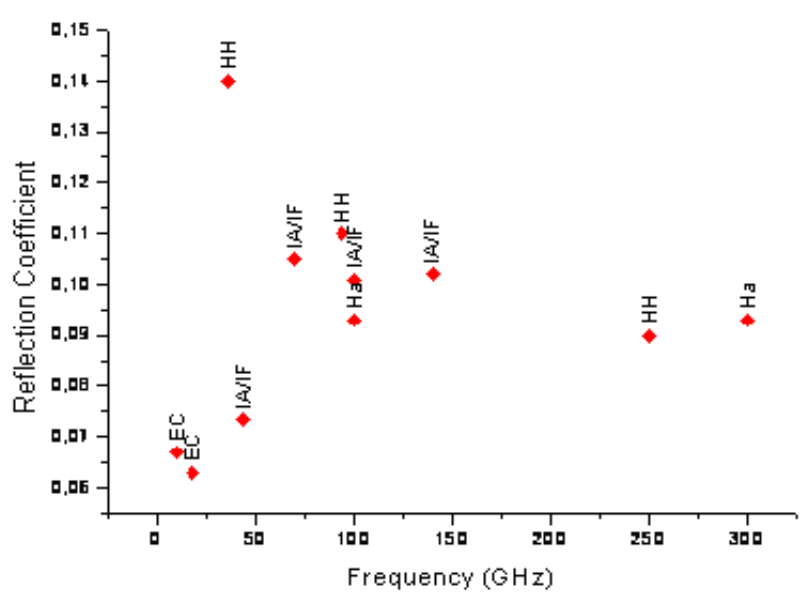

Fig. A.2. Reflection coefficient for ECCOSORB CR110. ECR: Emerson and Cumings, HH Hemmati et al. (1985), Ha: Halpern et al. (1986), IA/IF: this work

Table A.1. Aluminium 6061 - T6 Thermal properties at 4K (from CrioComp, Eckels Eng.)

\begin{tabular}{ll}
\hline \hline Thermal conductivity W/(m K) & 9.53 \\
Specific heat $\mathrm{J} /(\mathrm{Kg} \mathrm{K})$ & 0.28 \\
\hline
\end{tabular}

We report here some thermal and mechanical properties of the materials used in the $4 \mathrm{KRL}$ unit. Outgassing test, on samples prepared by the LFI team, were conducted by ESA.

Eccosorb and $\mathrm{Al}$ samples, bonded with epoxy adhesives, were submitted to lap-shear test (test run by ESA). Four epoxy resins were tested: Armstrong A-12, Loctite Hysol EA9394, Loctite 
Table A.4. Eccosorb CR-110 and CR-117 mechanical, thermal and outgassing properties. Data refer to 300K, unless where indicated

\begin{tabular}{lccc}
\hline \hline ECCOSORB $^{\text {TM }}$ & CR - 110 & CR - 117 & \\
& & & \\
Density $\left(\mathrm{g} / \mathrm{cm}^{3}\right)$ & 1.6 & 4.1 & $\begin{array}{c}\text { Emerson \& Cuming } \\
\text { this work }\end{array}$ \\
CTE (1/K) & $3 \times 10^{-5}$ & & this work \\
Elastic Mod. (MPa) & 3942 & 14336 & Halpern et al. (1986) \\
Thermal conductivity (W/(m K)) & 0.08 & & Peterson \& Richards $(1984)$ \\
Specific heat (J/(Kg K)) & $0.6 \mathrm{~T}^{2.05}$ & & Papendrecht $(2002 \mathrm{a})$ \\
Outgassing & & Papendrecht $(2002 \mathrm{a})$ \\
TML (\%) & 0.44 & & Papendrecht (2002a) \\
RML (\%) & 0.23 & & \\
CVCM (\%) & 0.0 & & \\
\hline
\end{tabular}

Table A.2. Electro-formed copper mechanical properties.

\begin{tabular}{lcc}
\hline \hline Elongation (\%) & 42.1 & this work \\
Elastic Modulus (GPa) & 115.1 & this work \\
Yield Strength (MPa) & 168 & this work \\
Ultimate Strength (MPa) & 269.4 & this work \\
\hline
\end{tabular}

Table A.3. Hysol EA 9394 Outgassing characteristics

\begin{tabular}{lll}
\hline \hline TML (\%) & 1.06 & Papendrecht \\
RML (2002b) & 0.34 & Papendrecht \\
CVCM (\%) & 0.01 & Papendrecht \\
$(2002 \mathrm{~b})$ \\
\hline
\end{tabular}
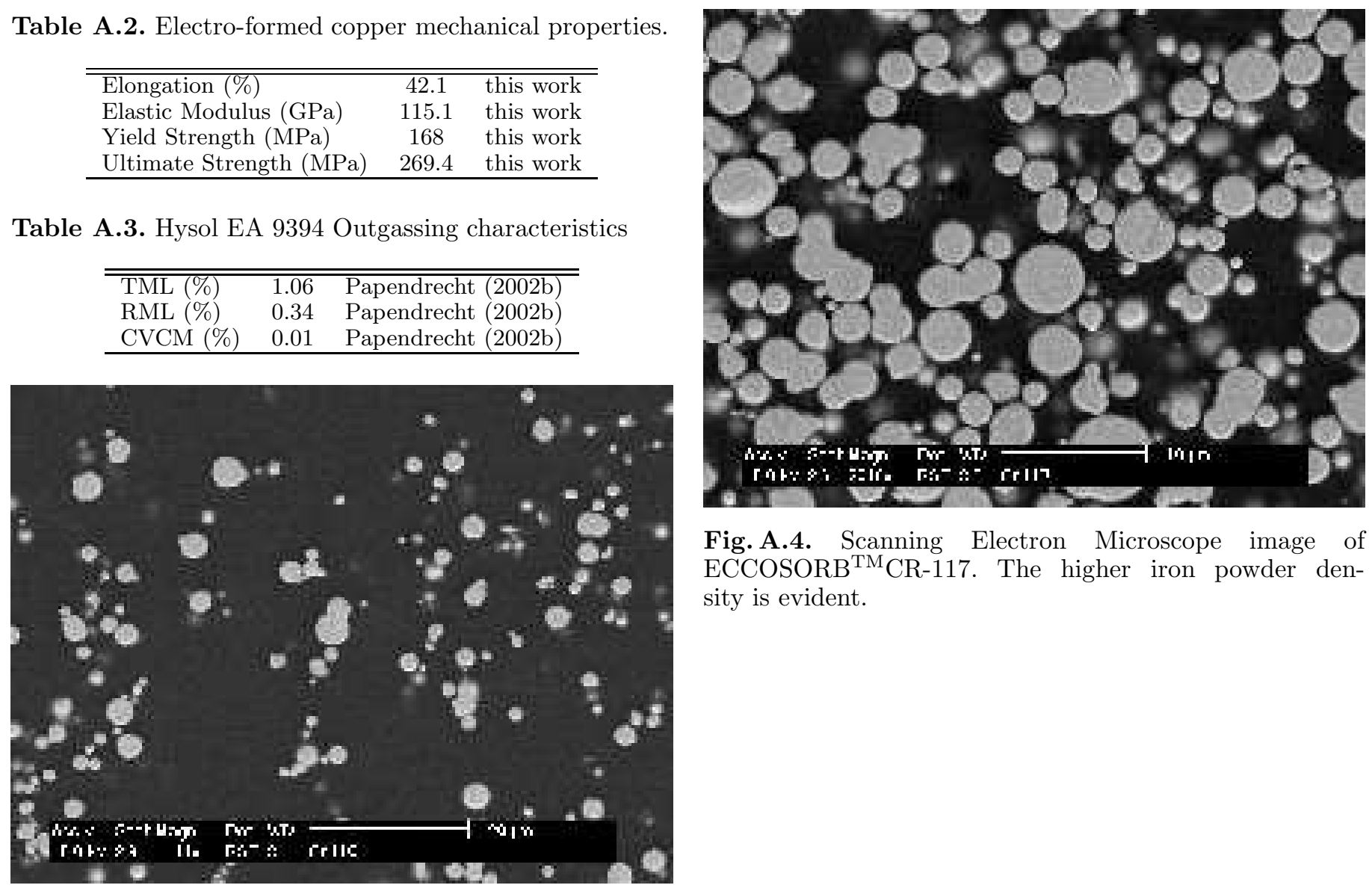

Fig. A.4. Scanning Electron Microscope image of ECCOSORB ${ }^{\mathrm{TM}} \mathrm{CR}-117$. The higher iron powder density is evident.

Fig. A.3. Scanning Electron Microscope image of ECCOSORB ${ }^{\mathrm{TM}} \mathrm{CR}-110$ obtained by the authors.

Hysol EA9361 and ECCOSORB CR110 (used as adhesive). Some samples had also been cycled 7 times at $77 \mathrm{~K}$. Results are reported in Table A.5.

Hysol EA9394 was selected for the superior performance. 
Table A.5. Mechanical properties of samples bonded with various epoxy adhesives. Data have been measured by ESA Material division.

\begin{tabular}{|c|c|c|c|c|c|c|}
\hline \multirow[t]{2}{*}{ Support } & \multirow[t]{2}{*}{ "Adhesive type } & \multicolumn{2}{|c|}{ Tensile stress $(\mathrm{MPa})$} & \multicolumn{2}{|c|}{ "Tensile strain (\%) } & \multirow[t]{2}{*}{ failure mode } \\
\hline & & cycled & not cycled & cycled & not cycled & \\
\hline CR-117/Al & $\bar{A}-12^{a}$ & 15.79 & 33.42 & 14.7 & 18.6 & in substrate \\
\hline CR-117/CR-110 & & 38.22 & 40.51 & 19.8 & 18.0 & in substrate \\
\hline $\mathrm{Al} / \mathrm{Al}$ & & - & 27.94 & - & 0.92 & in adhesive \\
\hline CR-117/Al & Hy9394 & 46.29 & 44.76 & 13.7 & 12.1 & in substrate \\
\hline CR-117/CR-110 & & 45.34 & 45.36 & 14.0 & 17.4 & in substrate \\
\hline $\mathrm{Al} / \mathrm{Al}$ & & - & 41.12 & - & 13.6 & in adhesive \\
\hline CR-110/Al & CR-110 & 7.05 & 21.4 & 4.7 & 8.2 & in adhesive \\
\hline CR-110/CR-110 & & 35.80 & 32.08 & 14.5 & 10.8 & in substrate \\
\hline CR-110/Al & $\mathrm{Hy} 9361^{d}$ & 23.47 & 22.21 & 8.3 & 7.6 & in adhesive \\
\hline CR-110/CR-110 & & 31.96 & 34.09 & 11.5 & 11.9 & in adhesive \\
\hline
\end{tabular}

\footnotetext{
${ }^{a}$ mixing ratio $1: 1$ in weight cured $6 \mathrm{~h}$ at $55 \mathrm{C}$

${ }^{b}$ mixing ratio 100:17 in weight, cured $2 \mathrm{~h}$ at $55 \mathrm{C}$

${ }^{c}$ mixing ratio $100: 12$ in weight, cured $4 \mathrm{~h}$ at $120 \mathrm{C}$

${ }^{d}$ mixing ratio $100: 1140$ in weight, cured $2 \mathrm{~h}$ at $65 \mathrm{C}$
} 